Richness, abundance and diversity of wild

THERYA, diciembre, 2013

mammals among habitats in the Sumidero

Vol.4(3):647-676

Canyon National Park, Chiapas, Mexico

DOI: 10.12933/therya-13-140

\title{
Riqueza, abundancia y diversidad de mamíferos silvestres entre hábitats en el Parque Nacional Cañón del Sumidero, Chiapas, México
}

\author{
Erika Arroyo Chacón ${ }^{1}$, Alejandra Riechers Pérez ${ }^{2 *}$, \\ Eduardo J. Naranjo ${ }^{3}$ y Gustavo Rivera-Velázquez ${ }^{4}$
}

\section{Abstract}

We assessed richness, abundance, and diversity (alpha and beta) of the wild mammals at the Cañon del Sumidero National Park, Chiapas, Mexico. Direct observations and captures of mammals were carried out in fragments of lowland and mid elevation evergreen tropical forest and seven habitats with different degrees of disturbance within the boundaries of the protected area. We recorded 77 mammal species, belonging to 10 orders, 24 families and 59 genera, with the following four species recorded as the most abundant in the study area: Artibeus jamaicensis, Peromycus mexicanus, Sturnira lilium and Liomys pictus. The mid or low elevation evergreen tropical forest had the highest species richness of mammals (26 species) and the highest alpha diversity $\left(\mathrm{H}^{\prime}\right.$ 2.52), but we did not find significant differences between the oak forest, the lowland mixed deciduous forest habitats with crops and fruit trees, and the disturbed area with pine trees, shade coffee plantations and pastures $\left(\mathrm{H}^{\prime} 2.51,2.37\right.$ and 2.29, respectively). For beta diversity, we found intermediate levels with the highest value split between the disturbed area with pastures, fallows and maize, vs. the disturbed area with pine trees, shade coffee plantations and pastures. The Cañon del Sumidero National Park is of great biological importance because it provides protection to native tropical forest communities in areas adjacent to the city of Tuxtla Gutierrez, sheltering a significant portion of the mammalian fauna that includes at least 11 threatened species listed in the Official Mexican Standard.

Keywords: Protected area, mammal fauna, Cañon del Sumidero, forests.

\section{Resumen}

Se estimó la riqueza y abundancia, así como la diversidad alfa y beta de los mamíferos silvestres del Parque Nacional Cañón del Sumidero, Chiapas, México. Para la

\footnotetext{
${ }^{1}$ Comisión Nacional de Áreas Naturales Protegidas. 2da. Oriente Norte 227, Palacio Federal, Colonia Centro, Tuxtla Gutiérrez, Chiapas, C. P. 29000. E-mail: arroyoe29@gmail.com (EAC)

${ }^{2}$ Coordinación Técnica de Investigación, Secretaría de Medio Ambiente e Historia Natural. Calzada de los Hombre Ilustres s/n, Col. Centro, Tuxtla Gutiérrez, Chiapas, C. P. 29000. Tel. Oficina 019616000254 ext. 104. E-mail: alexriechers@ yahoo.com.mx (ARP)

${ }^{3}$ El Colegio de la Frontera Sur, Unidad San Cristóbal de Las Casas. E-mail: enaranjo@ecosur.mx (EJN)

${ }^{4}$ Facultad de Ciencias Biológicas, Universidad de Ciencias y Artes de Chiapas.

* Corresponding author.
} 
observación y captura de mamíferos se realizaron muestreos en un fragmento de selva mediana o baja perennifolia y siete hábitats con diferente grado de perturbación en el área natural protegida. Se registraron 77 especies de mamíferos silvestres pertenecientes a 10 órdenes, 24 familias y 59 géneros, siendo las especies más abundantes Artibeus jamaicensis, Peromycus mexicanus, Sturnira lilium y Liomys pictus. La selva mediana o baja perennifolia presentó la mayor riqueza de especies de mamíferos (26 especies) y la mayor diversidad alfa $\left(H^{\prime} 2,52\right)$, pero no se encontraron diferencias significativas con el encinar, la selva baja caducifolia con cultivos y árboles frutales, y el área perturbada con pinos, cafetales de sombra y potreros $\left(\mathrm{H}^{\prime} 2.51,2.37\right.$ y 2.29 , respectivamente). La diversidad beta fue intermedia, el valor más alto fue en el área perturbada con potreros, acahuales y cultivos de maíz vs. el área perturbada con pinos, cafetales de sombra y potreros. El Parque Nacional Cañón del Sumidero es de gran importancia biológica porque contiene áreas de selvas conservadas, adyacentes a la zona urbana de la Ciudad de Tuxtla Gutiérrez, las cuales albergan una importante riqueza mastofaunística en donde se incluyen 11 especies en alguna categoría de riesgo de acuerdo a la Norma Oficial Mexicana.

Palabras clave: Área natural protegida, Mastofauna, Cañón del Sumidero, Selvas.

Introducción

La diversidad biológica (biodiversidad) se entiende como la variabilidad de organismos vivos, presentes en los distintos hábitats de ecosistemas terrestres y acuáticos, a diferentes escalas biológicas (UNEP 1992). Aún cuando el término ha sido cuestionado, así como la forma de medirlo (Hurlbert 1971); actualmente ha tomado gran importancia y validez.

De hecho se ha desarrollado un número importante de cuantificaciones sobre la diversidad de especies mediante la separación de sus componentes alfa, beta y gamma, como indicadores del estado de conservación de los ecosistemas y con aplicabilidad para fines de manejo y monitoreo ambiental (Whittaker 1972; Spellerberg 1991; Halffter y Moreno 2005).

La diversidad alfa se define como el número de especies de una comunidad; la diversidad beta, como la variación en el número de especies que se produce entre un hábitat y otro, o también definido como el grado de recambio o reemplazo en la composición de especies entre diferentes comunidades en un paisaje, y la diversidad gamma como el número de especies del conjunto de comunidades que integran un paisaje, que resulta de la diversidad alfa y beta (Whittaker 1972; Moreno 2001). La diversidad del paisaje no es simplemente la adición de los valores de la diversidad de hábitats, sino que está determinada por la relación entre la variación espacial y temporal de cada hábitat y el reemplazo espacial de especies entre éstos (Koleff 2005). Los valores de diversidad son comúnmente obtenidos mediante índices, que reflejan el número total de especies, en particular de un taxón y en un sitio específico; pero algunos índices se han basado en la riqueza de especies y no consideran la abundancia de las especies (Pianka 1966).

El estado de Chiapas, ubicado en el sur de México, ocupa el primer lugar a nivel nacional en riqueza de mamíferos terrestres silvestres con 205 especies, 117 géneros y 31 familias; siete de estas especies son endémicas del estado, y los grupos mejor 
representados son los quirópteros, los roedores y los carnívoros (Retana y Lorenzo 2002; Naranjo et al. 2005). Muchas de estas especies de mamíferos silvestres se encuentran dentro de áreas naturales protegidas, pero solo en algunas se han realizado estudios sobre la riqueza, abundancia y diversidad de las especies de este grupo.

El Parque Nacional Cañón del Sumidero es un área natural protegida federal considerada como un elemento importante del patrimonio natural de México por su belleza escénica, ha sufrido fuertes problemas de asentamientos humanos irregulares que han provocado cambios de uso del suelo y transformación de la vegetación nativa. En Chiapas se desconoce la situación de riesgo de numerosas especies de mamíferos silvestres, lo que se acentúa por los procesos de pérdida de hábitat y por la cacería indiscriminada, provocando cambios profundos en la composición de especies y las abundancias de sus poblaciones (Naranjo 2002; Naranjo et al. 2005).

El Parque Nacional Cañón del Sumidero fue decretado como área natural protegida desde 1980, en 1990 se realizaron los primeros inventarios faunísticos del área (GálvezAltúzar 1990; Ovando-Damián 1990), por ello en el 2007 surge la necesidad de conocer y actualizar el listado mastofaunístico del Parque, proyecto financiado por la Comisión Nacional para el Conocimiento y Uso de la Biodiversidad (Altamirano-González et al. 2007), del cual se deriva el presente documento.

Específicamente, en este trabajo se analizan la riqueza, abundancia, diversidad alfa y beta de los mamíferos silvestres en un fragmento de selva mediana o baja perennifolia con siete hábitats con diferente grado de perturbación, con el objetivo de obtener información actualizada que sirva de base para las políticas del programa de conservación y manejo del Parque.

\section{Material}

y Métodos

Área de estudio. El Parque Nacional Cañón del Sumidero se ubica al centro-oeste del estado de Chiapas, en los límites de tres regiones fisiográficas, la Depresión Central, las Montañas del Norte y los Altos de Chiapas. Abarca los municipios de Chiapa de Corzo, Soyaló, Osumacinta, San Fernando y Tuxtla Gutiérrez (Fig. 1), con una superficie de 21,789 ha. Sus coordenadas extremas se ubican entre $16^{\circ} 44^{\prime}$ y $16^{\circ} 56^{\prime}$ de Latitud Norte y $-93^{\circ} 00^{\prime}$ y $-93^{\circ} 11^{\prime}$ de Longitud Oeste, con una altitud que va desde los 360 a 1,720 m (López-Portillo 1980). Los tipos de vegetación presentes en el Parque son: selva baja caducifolia, selva alta o mediana subperennifolia, selva mediana o baja perennifolia, bosque de pino y bosque de encino principalmente (Miranda y Hernández X. 1963; Miranda 1998).

Dentro del Parque Nacional Cañón del Sumidero existen asentamientos humanos, previos a la creación del área natural protegida, en su mayoría comunidades y rancherías, muchas de ellas de origen Tzeltal, Tzotzil y Zoque, que se dedican principalmente a la agricultura y ganadería (PRONATURA-Chiapas A. C. 2002).

Hábitats muestreados. Se seleccionaron ocho hábitats de muestreo dentro del Parque Nacional (Fig. 1), mediante un Sistema de Información Geográfica (SIG), reconociéndose en total cuatro tipos de vegetación: encinar, selva alta o mediana subperennifolia, selva mediana o baja perennifolia y selva baja caducifolia, considerando la clasificación propuesta por Miranda y Hernández X. (1963). Con el apoyo del equipo técnico de la 
Comisión Nacional de Áreas Naturales Protegidas (CONANP) se solicitó el consentimiento de los pobladores de las comunidades asentadas dentro del área protegida para realizar los muestreos de mamíferos. Los ocho hábitats se distribuyen (Fig. 1) como sigue:

1. Encinar (ENC): ubicado en el sitio "Kilómetro 12", que se localiza al costado izquierdo del kilómetro 12 de la carretera federal Tuxtla Gutiérrez-Cañón del Sumidero, en el municipio de Tuxtla Gutiérrez ( $16^{\circ} 48^{\prime} 50^{\prime \prime} \mathrm{N}$ y $-93^{\circ} 06^{\prime} 07.2^{\prime \prime} \mathrm{W}$ ), a una altitud de 1,243 m. El hábitat se clasificó como encinar, ya que está constituido por Quercus sp.

2. Selva alta o mediana subperennifolia con cafetales de sombra (SA): ubicado en el sitio "Tierra Colorada", que se localiza a un costado de la población Tierra Colorada, en el municipio de Tuxtla Gutiérrez ( $16^{\circ} 50^{\prime} 26,4^{\prime \prime} \mathrm{N}$ y $-93^{\circ} 07^{\prime} 56.5^{\prime \prime} \mathrm{W}$ ), a 1,240 m de altitud. El tipo de vegetación es selva alta o mediana subperennifolia, también se encuentran inmersos en ella cultivos de café orgánico de sombra bajo, un sistema de policultivo tradicional.

3. Selva mediana o baja perennifolia (SM): ubicado en el sitio "Mirador La Atalaya o Los Chiapa", que se localiza al final de la carretera federal Tuxtla Gutiérrez-Cañón del Sumidero, en el municipio de Tuxtla Gutiérrez (Kilómetro 22) a una altitud de 1,258

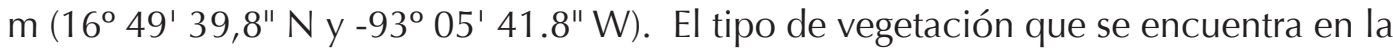
parte sur de este sitio es selva mediana o baja subperennifolia y en la parte norte es selva alta o mediana perennifolia; denominando a este hábitat como selva mediana a baja subperennifolia, porque en él se muestreo la mastofauna.

4. Selva baja caducifolia (SBC1): ubicado en el sitio "Predio El Jardín", se encuentra al margen noreste del río Grijalva a una altitud de 390 m (16 $16^{\circ} 53^{\prime}$ 8,6" N y -93 06' 2.2" W), es un área turística y pertenece al municipio de Osumacinta.

5. Selva baja caducifolia con cultivos (frijol y maíz) y árboles frutales (SBC2): ubicado en el sitio "Rancho Magaly", se encuentra al margen suroeste del río Grijalva $\left(16^{\circ}\right.$ $45^{\prime} 33,8^{\prime \prime} \mathrm{N} \mathrm{y}-93^{\circ} 02^{\prime} 32,2^{\prime \prime} \mathrm{W}$ ), se ubica en el municipio de Chiapa de Corzo a una altitud de $425 \mathrm{~m}$; anteriormente era una propiedad privada. El tipo de vegetación es selva baja caducifolia, mientras que en el área rivereña se encuentran pequeños cultivos de frijol (Phaseolus vulgaris), papaya (Carica papaya) y maíz (Zea maíz), así como árboles frutales de mango (Mangifera indica), capulín (Muntingia calabura), plátano (Musa sp.), papausa (Anona diversifolia), entre otros.

6. Área Perturbada con selva alta a mediana subperennifolia, potreros, acahuales y cultivo de maíz (AP1): ubicado en el sitio "Predio Tzanet" pertenece al municipio de

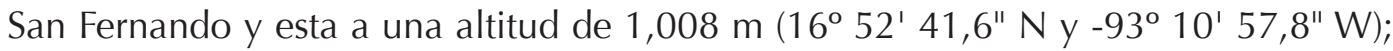
se clasifico como área perturbada porque, a pesar de presentar fragmentos de selva alta a mediana subperennifolia, está fuertemente afectado por potreros, acahuales jóvenes y cultivos de maíz, una parcela de maíz presentaba árboles frutales como plátano (Musa sp.), guayaba (Psidium guajava) y limón (Citrus sp.).

7. Área Perturbada con pinos, cafetales de sombra y potreros (AP2): ubicado en el sitio "Ranchería Agua Fría", pertenece al municipio de Osumacinta, a una altitud de 1,197

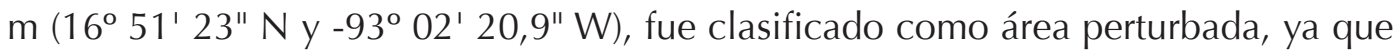
presenta parches de pino con cultivos de café de sombra y áreas de potreros.

8. Área Perturbada con encinares, con selva alta o mediana subperennifolia y pastizal de zacate estrella (AP3): ubicado en el sitio "Rancho El Alteño", pertenece al municipio 
de San Fernando, a una altitud de 1,200 m (16 $55^{\prime} 21,0^{\prime \prime} \mathrm{N}$ y $\left.-93^{\circ} 10^{\prime} 17^{\prime \prime} \mathrm{W}\right)$; es propiedad privada dedicada a la ganadería; se clasificó como área perturba, porque a pesar de encontrar manchones de encinares (Quercus sp.) y al margen del arroyo árboles de selva alta o mediana subperennifolia, el área está fuertemente modificada por pastizales de zacate estrella (Cynodon plectostachyus) para el consumo por ganado.
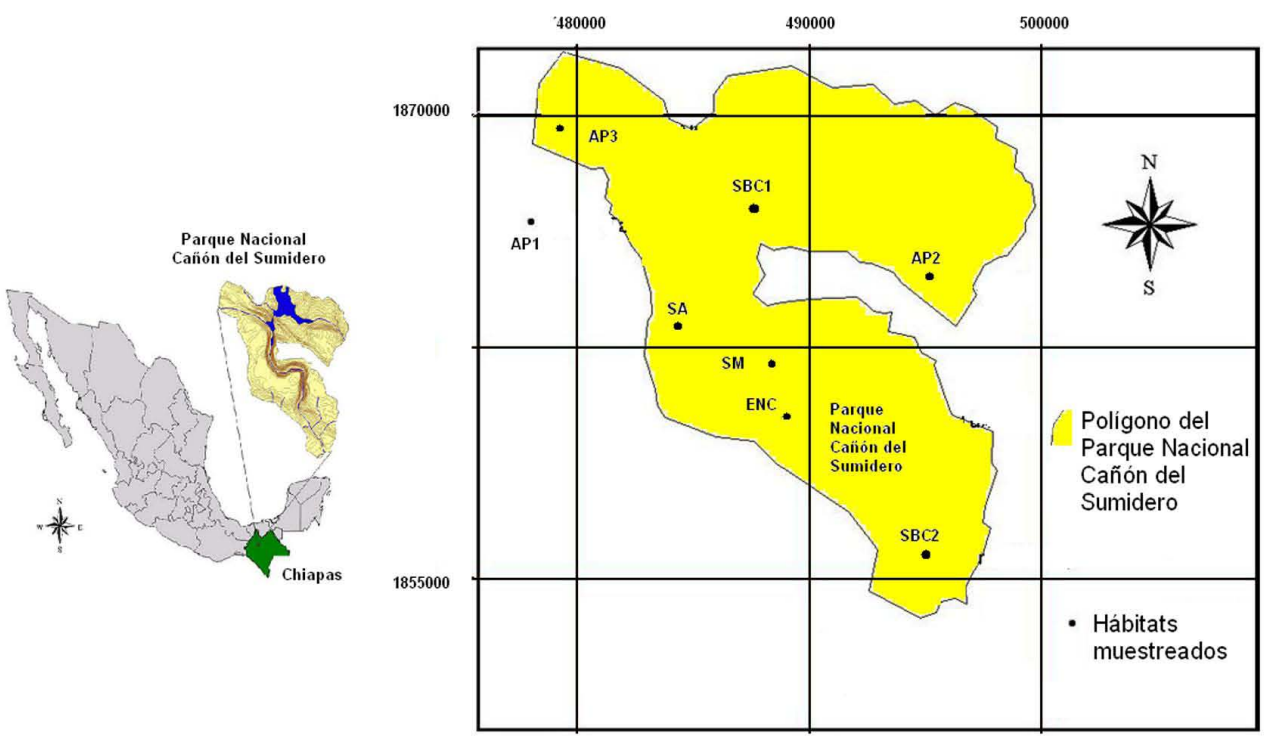

Figura 1. Localización de los hábitats muestreados en el Parque Nacional Cañón del Sumidero, Chiapas, México.

Abreviatura para el hábitat: ENC, encinar; SA, selva alta o mediana subperennifolia con cafetales de sombra; SM, selva mediana o baja perennifolia; SBC1, selva baja caducifolia; SBC2, selva baja caducifolia con cultivos (frijol y maíz) y árboles frutales; AP1, área perturbada con selva alta o mediana subperennifolia, potreros, acahuales $y$ cultivos de maíz; AP2, área perturbada con pinos, cafetales de sombra y potreros; AP3, área perturbada con encinares, con selva alta o mediana subperennifolia y pastizal de zacate estrella.

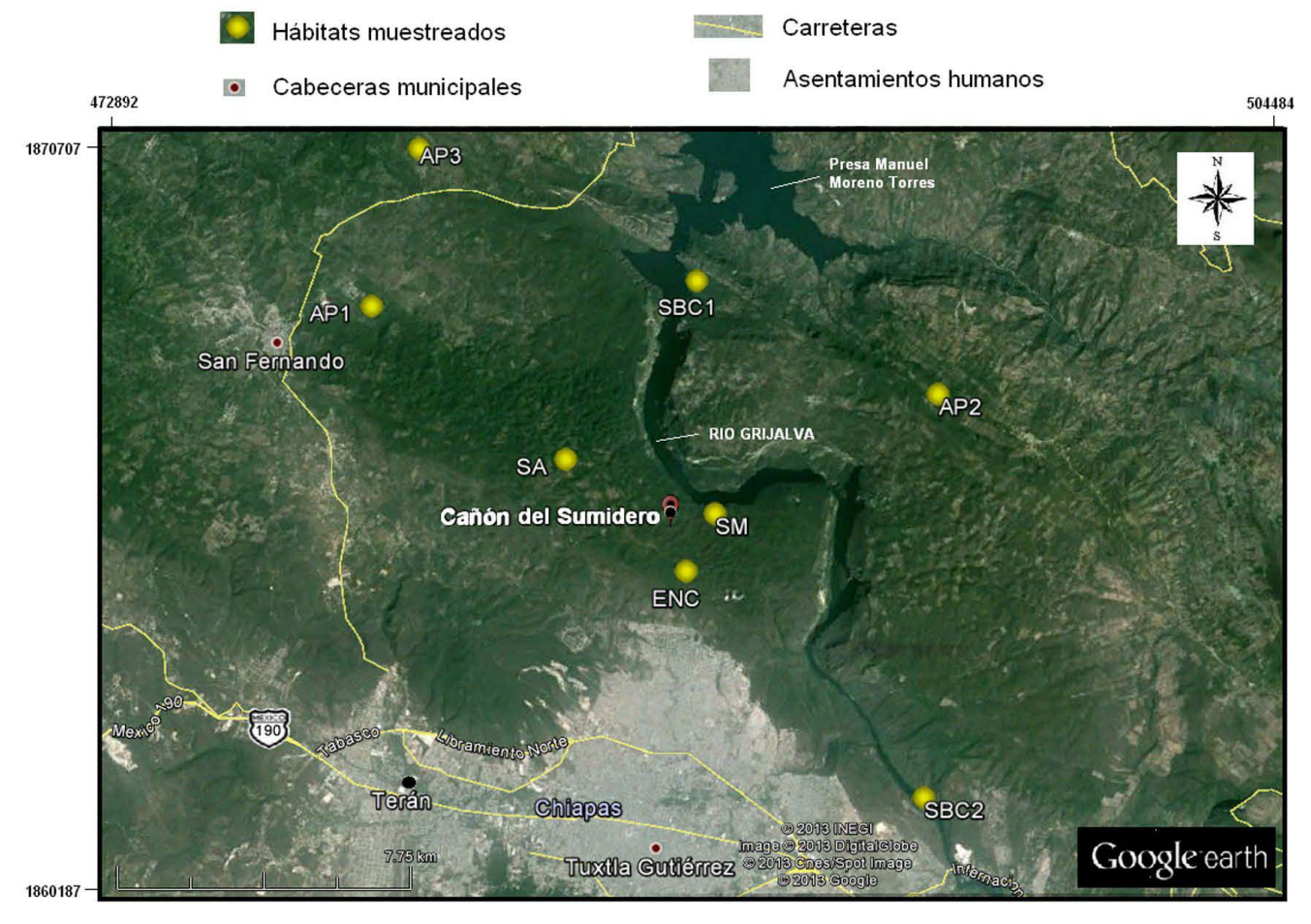

Consulta de bases de datos de colecciones científicas y revisión de literatura especializada. Para completar el listado de especies de mamíferos del Parque Nacional Cañón del Sumidero, se consultaron las bases de datos del Museo de Zoología Alfonso L. Herrera 
(MZFC) de la Facultad de Ciencias de la Universidad Nacional Autónoma de México; la Colección de Mamíferos de El Colegio de la Frontera Sur Unidad San Cristóbal de las Casas (ECO-SC-M); el Museo de Zoología de la Universidad de Ciencias y Artes de Chiapas (MZICACH) y la Colección Zoológica Regional Mammalia (CZRMA) de la Secretaría del Medio Ambiente e Historia Natural (SEMAHN) de Gobierno del Estado de Chiapas. En esta última colección (CZRMA), además de la consulta de la base de datos, también se examinaron los ejemplares y se verificó la determinación taxonómica.

También se consultó literatura (Gálvez-Altúzar 1990; Riechers y De la Cruz 2012) con registros de mamíferos colectados en localidades de Chiapas, seleccionando los registros provenientes del Parque Nacional Cañón del Sumidero.

Trabajo de campo. El registro de mamíferos silvestres se realizó mensualmente a partir de junio de 2004 a noviembre de 2005. Cada hábitat (Fig.1) se visitó dos veces durante el período de muestreo con una duración de cinco días por visita. Para la captura de roedores se colocaron diariamente 75 trampas Sherman plegables de $8 \times 9$ x $23 \mathrm{~cm}$, en transectos de longitud variable con una distancia regular de cinco metros entre cada trampa. Cada transecto se realizaba en un lugar diferente por día, con la finalidad de abarcar varios microhábitats, incluyendo la vegetación nativa, los cultivos, las madrigueras y lugares rocosos. Las trampas se cebaron con una mezcla de avena esencia de vainilla y se colocaron a partir de las 16:00 horas, levantándose durante la mañana del día siguiente. Para la captura de murciélagos, se utilizaron tres redes de hilo nylon de 6 × 2 m durante cada noche de muestreo, las cuales se colocaron dentro de la vegetación, en caminos, en cuerpos de agua y en refugios. Las redes se abrieron de las 19:00 horas hasta las 00:00 horas, realizándose revisiones cada hora.

Para el registro y captura de mamíferos medianos se utilizaron cuatro trampas de alambre Tomahawk por día, cebadas con sardina, colocándolas en senderos, madrigueras, cerca de cuerpos de agua o donde se registraran huellas, siendo revisadas diariamente. Además, para la observación directa de ejemplares se realizaron recorridos diurnos (7:00 - 9:00 y 16:00 - 18:00 horas) y nocturnos (19:00 - 00:00 horas). Los recorridos se efectuaron a lo largo de trayectos con una extensión de uno a tres kilómetros; considerando los registros auditivos cuando se escucharon las vocalizaciones de mamíferos, también se realizaron observaciones de excretas, madrigueras, restos de huesos, pelos y huellas, obteniéndose la impresión de estas últimas con una mezcla de yeso odontológico. Los rastros de mamíferos fueron identificados con ayuda de guías de campo (Aranda 2000). Para complementar la riqueza de especies de mamíferos silvestres, se realizaron entrevistas no estructuradas a pobladores locales con la ayuda de dibujos tomados de la Guía de Mamíferos Silvestres de Chiapas (Aranda y March 1987).

Los ejemplares que se capturaron en redes o trampas fueron liberados en el lugar de captura posteriormente a su identificación específica. Se colectaron cinco ejemplares por especie capturada, siempre y cuando está no estuviera incluida en la Norma Oficial Mexicana NOM-059-SEMARNAT-2010 (Diario Oficial de la Federación 2010), de acuerdo a lo estipulado en el Permiso Especial de Colecta Científica (Oficio Núm. SGPA/DGVS/07782) emitido el 20 de agosto del 2004 por la Dirección General de Vida Silvestre de la Secretaría de Medio Ambiente y Recurso Naturales. A los especímenes recolectados se les registraron las medidas externas básicas (longitud total, longitud 
de la cola, longitud de la pata posterior izquierda, longitud de la oreja y longitud del antebrazo en el caso de murciélagos) y el peso; su condición reproductiva en machos fue determinada por la posición de los testículos (reproductivos y no reproductivos); en hembras por la palpación del vientre y la observación de las mamas (no reproductivas, preñadas, lactantes y postlactantes; Racey 1988); la categoría de edad (juvenil, subadulto y adulto) fue determinada por la observación de la fusión de la epífisis-diáfisis de la cuarta articulación metacarpal-falangeal para los murciélagos (Anthony 1988) y por coloración del pelaje y desgaste de molares en roedores. Posteriormente, se procedió a prepararlos como piel y cráneo o en alcohol al 70\%, de acuerdo a técnicas convencionales para preservación de material científico (Llorente et al. 1990). Los ejemplares recolectados fueron depositados en la Colección Zoológica Regional Mammalia (CZRMA) de la Secretaría de Medio Ambiente e Historia Natural (SEMAHN) del Gobierno del Estado de Chiapas o en la Colección de Mamíferos de El Colegio de la Frontera Sur San Cristóbal de las Casas (ECO-SC-M). La determinación de los ejemplares se hizo a nivel de especie con el apoyo de descripciones originales, monografías y claves especializadas (Álvarez et al. 1994; Hall 1981; Medellín et al. 1997). Se utilizó la nomenclatura taxonómica propuesta por Ramírez-Pulido et al. (2005b).

Análisis de la información. La riqueza de especies se obtuvo mediante el conteo de especies presentes en cada hábitat, así como de los registros citados en literatura y la consulta de colecciones científicas locales y nacionales. El esfuerzo de captura para murciélagos se calculó según el método de Medellín (1993), considerando el número de metros lineales $(\mathrm{m})$ de red y las horas (hrs.) que estas permanecieron abiertas durante el período de muestreo. Para el resto de los pequeños y medianos mamíferos, el esfuerzo se calculó con el número de trampas Sherman y Tomahawk colocadas en cada hábitat, multiplicado por los días muestreo.

Para obtener la eficiencia del muestreo en el registro de mamíferos se aplicaron los criterios señalados por Clench (1979), obteniéndose curvas de incremento acumulativo de especies a través del tiempo (Soberón y Llorente 1993), mediante la siguiente fórmula: en donde $\mathrm{S}(\mathrm{t})=$ at $/(1+\mathrm{bt})$, donde: $\mathrm{S}(\mathrm{t})=$ número de especies esperadas en el tiempo, $\mathrm{t}$ = medida del esfuerzo en función del tiempo, $\mathrm{a}=$ tasa de incremento al comienzo del muestreo, $b=$ acumulación de especies. Este índice se calculó solamente con la riqueza y abundancia de especies registradas directamente en campo; no se consideraron los registros obtenidos de colecciones científicas ni de la literatura.

La abundancia relativa de cada especie se calculó mediante la ecuación: $A R=S / N X$ 100, donde: $\mathrm{AR}=$ abundancia relativa de la especie $i, \mathrm{~S}=$ número total de individuos de la especie $i, \mathrm{~N}=$ número total de individuos registrados en el área de estudio (Naranjo 2000). El estado actual de conservación de cada especie se consultó en la Norma Oficial Mexicana NOM-059-SEMARNAT-2010 (Diario Oficial de la Federación 2010) que considera la categorías: E: probablemente extinta en el medio silvestre, P: en peligro de extinción, A: Amenazadas y Pr: Sujetas a protección especial.

Para estimar la diversidad alfa de los hábitats se utilizó el índice de diversidad de Shannon - Wiener, mediante la ecuación: $\mathrm{H}^{\prime}=-\Sigma P i l n$ Pi, en donde: $P i=$ proporción de individuos registrados de la i-ésima especie (Magurran 1989; Moreno 2001). Para determinar diferencias estadísticas en la diversidad alfa entre los hábitats, se aplicó una 
prueba de $t$ de student modificada por Hutchenson (Brower et al. 1997). La diversidad beta entre dos muestras se calculó por sustracción del valor de similitud a la unidad: $\mathrm{d}=1-\mathrm{s}$, en donde $\mathrm{d}=$ disimilitud y $\mathrm{s}=$ similitud; $\mathrm{s}$ se obtuvo mediante el coeficiente de Sorensen para datos cualitativos: $\mathrm{Cs}=2 j$ / $(\mathrm{a}+\mathrm{b})$, en donde: $j=$ número de especies compartidas entre ambas localidades, $\mathrm{a}=$ número de especies en la localidad $\mathrm{A}, \mathrm{b}=$ número de especies en la localidad B (Magurran 1989).

Resultados y

Discusión

Esfuerzo de muestreo. El esfuerzo de captura fue de 368,640 m red/hora para murciélagos, de 388,800 trampas/noche para los mamíferos pequeños no voladores y de 16,384 trampas/noche para mamíferos medianos. La curva de Clench presentó un comportamiento asintótico y permitió registrar el $86.17 \%$ de las especies estimadas (53 especies observadas de las 61.5 esperadas) con un $99.2 \%$ de confiabilidad en el muestreo (Fig. 2), sugiriendo que el esfuerzo de muestreo realizado en campo fue idóneo.

Riqueza de especies / Composición de especies. Se registraron 77 especies de mamíferos silvestres en el Parque Nacional Cañón del Sumidero (Tabla 1). De este total, 40 especies fueron detectadas mediante captura en el campo; 39, a través de consultas a colecciones científicas; 28, mediante entrevistas con pobladores locales y 26 por consulta de literatura especializada, cabe mencionar que 44 especies presentaron más de un tipo de registro (Tabla 1). Durante el trabajo de campo se obtuvieron 1089 registros de ejemplares, de los cuales el $11.75 \%(n=128)$ correspondió a observaciones directas, $76.77 \%(836)$ a capturas, $8.17 \%$ (89) a colectas, $1.84 \%$ (20) a observación de excretas, $0.92 \%$ (10) a huellas y $0.55 \%$ (6) a registros auditivos.

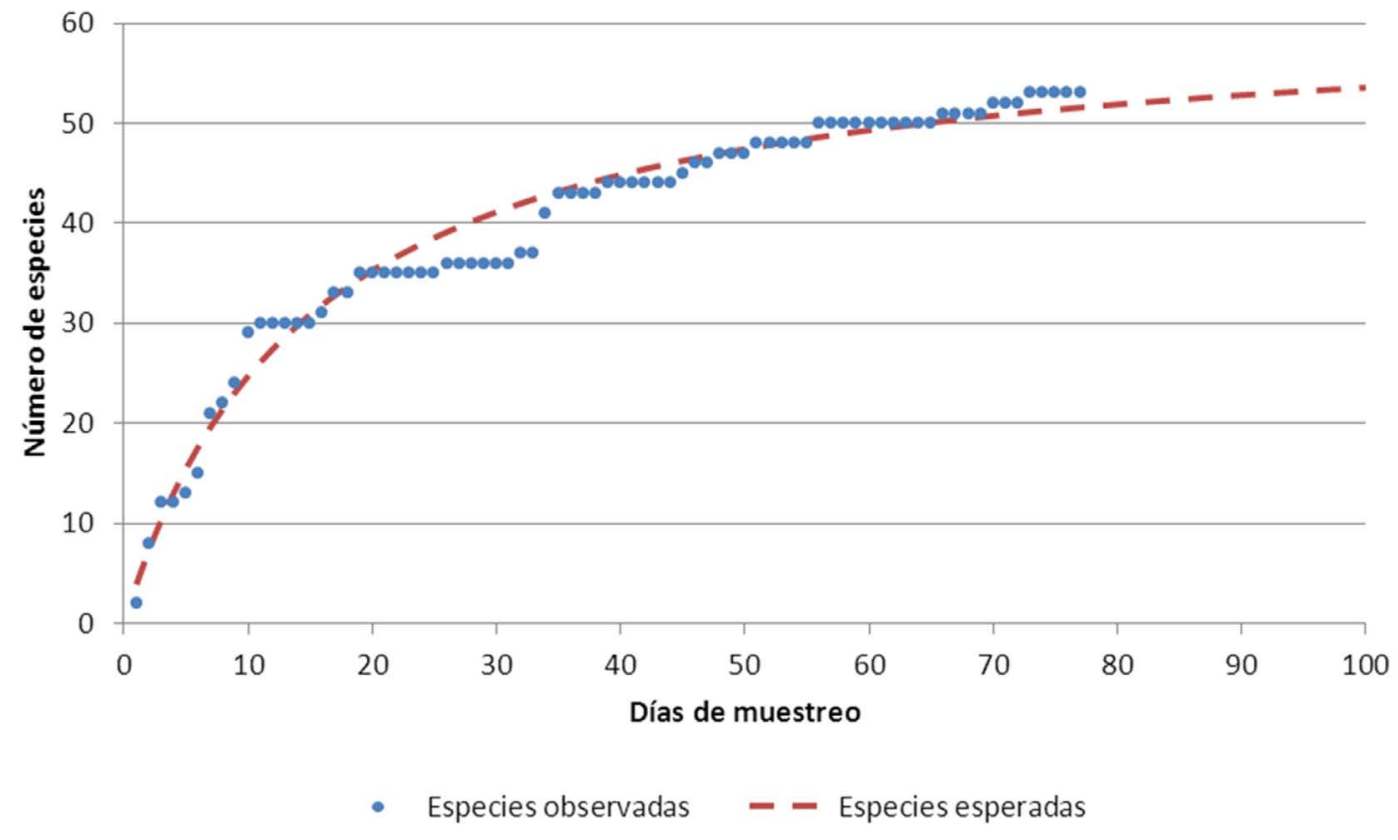

Figura 2. Curva de acumulación de especies de Clench, mamíferos registrados durante junio del 2004 a noviembre del 2005 en el Parque Nacional Cañón del Sumidero, Chiapas.

Las 77 especies de mamíferos registradas en el área de estudio pertenecen a 10 órdenes, 24 familias y 59 géneros (Fig. 3), representando el 14.7\% de la mastofauna mexicana (525 especies, Ceballos et al. 2005) y el 37.75\% de los mamíferos terrestres de Chiapas (205 especies; Naranjo et al. 2005). 
Tabla 1. Lista taxonómica, tipo de registro $y$ categoría de riesgo de los mamíferos silvestres en el Parque Nacional Cañón del Sumidero, Chiapas. Nomenclatura taxonómica de acuerdo a Ramírez-Pulido et al. $2005 b$.

\section{ORDEN \\ Familia Subfamilia \\ Tribu \\ DIDELPHIMORPHIA \\ Didelphidae \\ Didelphinae \\ Monodelphini}

ESPECIE

ER BK003

ER BCC

CR

Marmosa mexicana Merriam, 1897

Didelphis marsupialis

Linnaeus, 1758

EN

Didelphis virginiana

Kerr, 1792

2383 CZRMA

C,O,EN,LT,CC 2511 CZRMA 314,315MZ-ICACH

\section{CINGULATA \\ Dasypodidae \\ Dasypodinae}

Dasypodini

Dasypus

novemcinctus

Linnaeus, 1758

PILOSA

Myrmecophagidae

\section{LAGOMORPHA}

Leporidae

\section{RODENTIA \\ Sciuridae \\ Sciurinae}

Sciurini

Sciurus aureogaster F. Cuvier, 1829

Sciurus deppei Peters, 1863

Tamandua mexicana (de Saussure, 1860)

Sylvilagus floridanus (J. A. Allen, 1890)

$\mathrm{O}, \mathrm{EN}, \mathrm{CC}$

$632 \mathrm{MZ}-\mathrm{ICACH}$

1201 MZ-ICACH
2324,2325

Baiomys musculus (Merriam, 1892)

Tylomys nudicaudus (Peters, 1866)

Neotomini

Neotoma mexicana Baird, 1855

Peromyscus aztecus (de Saussure, 1860)

Peromyscus levipes Merriam, 1898

Peromyscus melanophrys (Coues, 1874)

Peromyscus mexicanus (de Saussure, 1860)
C,LT

2347 CZRMA

C,L

2434 CZRMA

C,LT

2348 CZRMA
2349,2326,
C,L,O,CC,LT $\quad 2327,2392$
2634,2657 CZRMA
2352 CZRMA

2346 CZRMA

1412 MZ-ICACH
540 ECO-SC-M;

$5,32,33,35,40,41$,

65,66,213,288

MZ-ICACH;

2628,2629,2640,

2644 CZRMA 
Continúa Tabla 1 ...

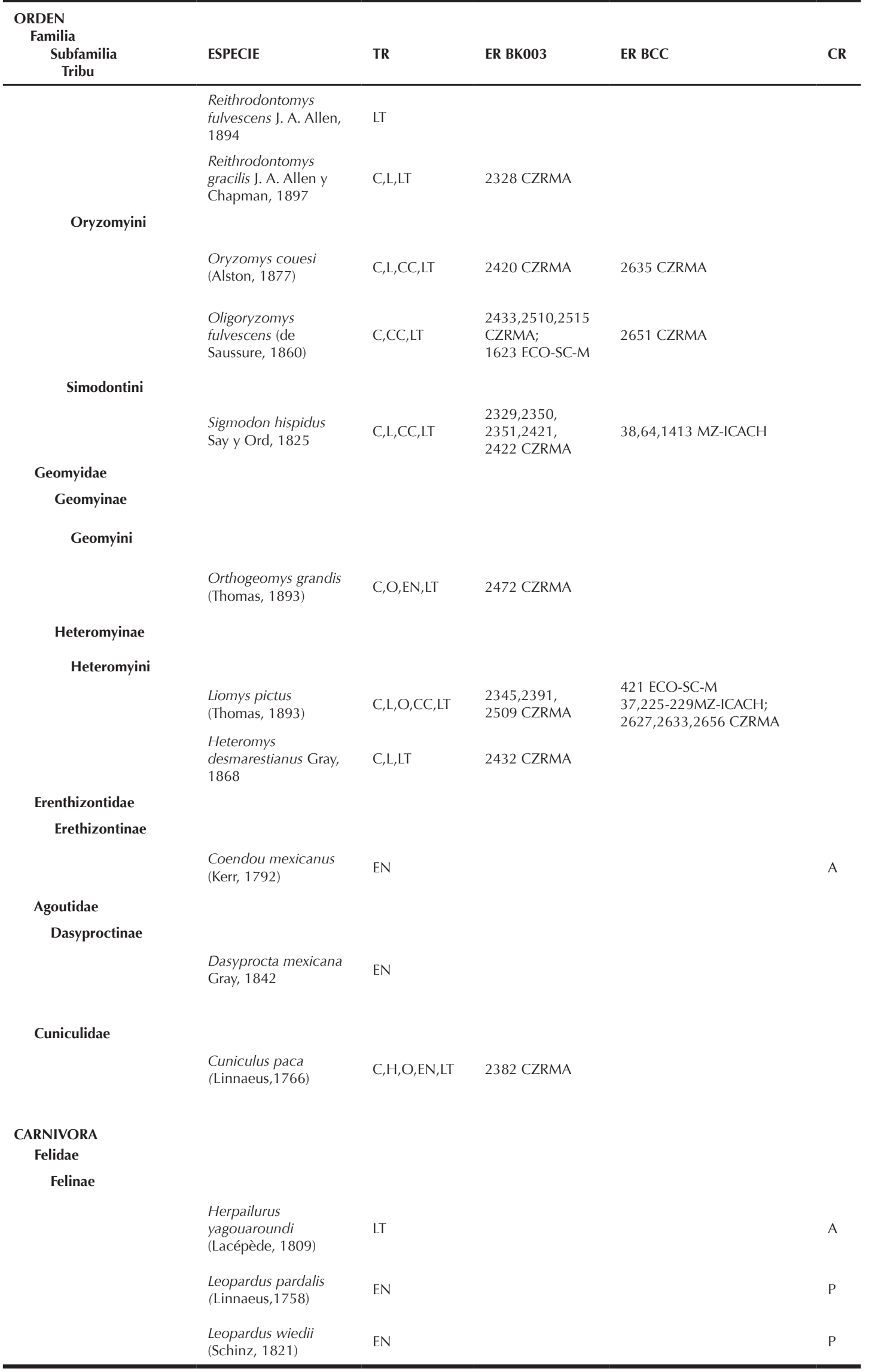




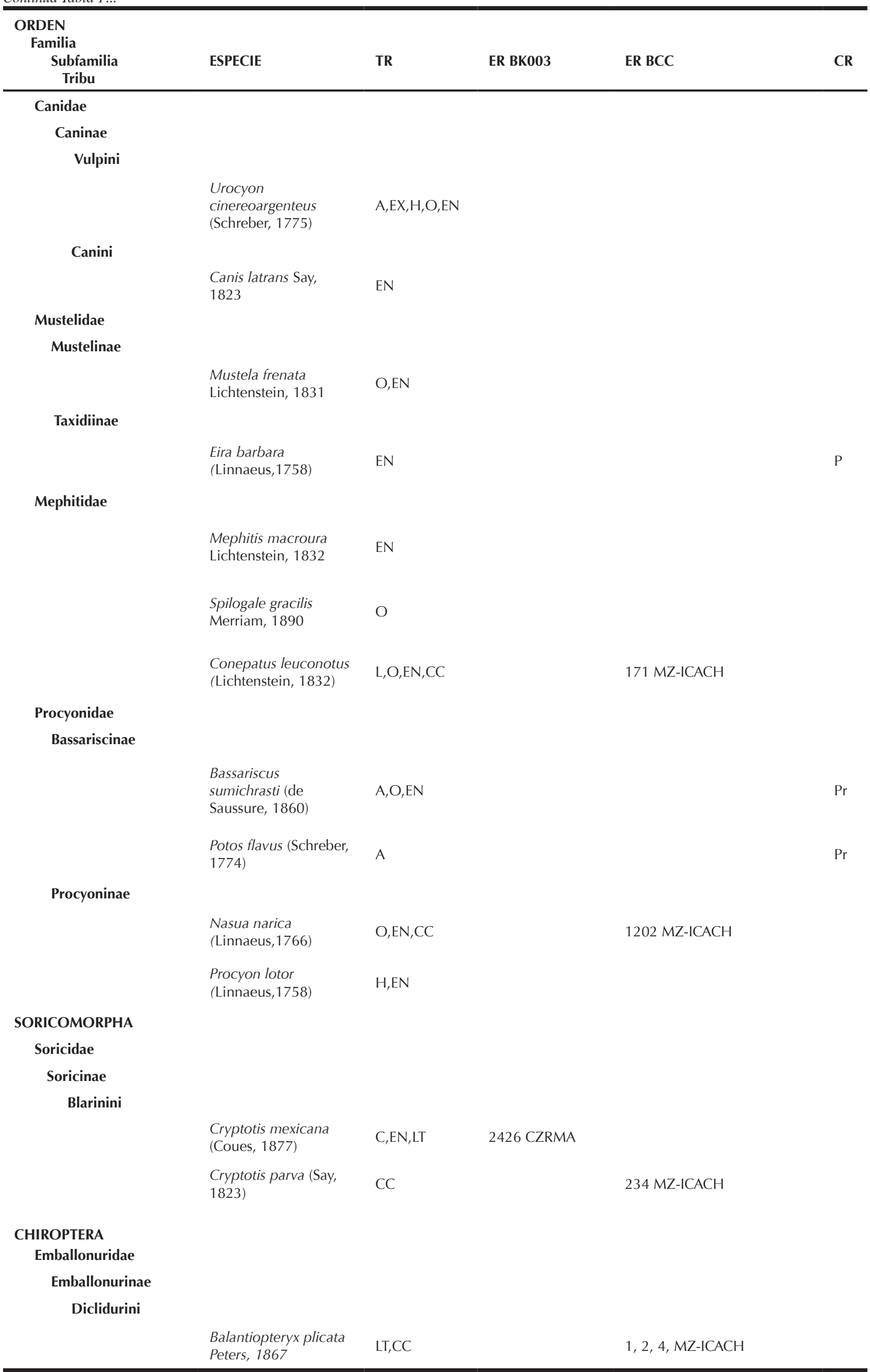


Continúa Tabla 1...

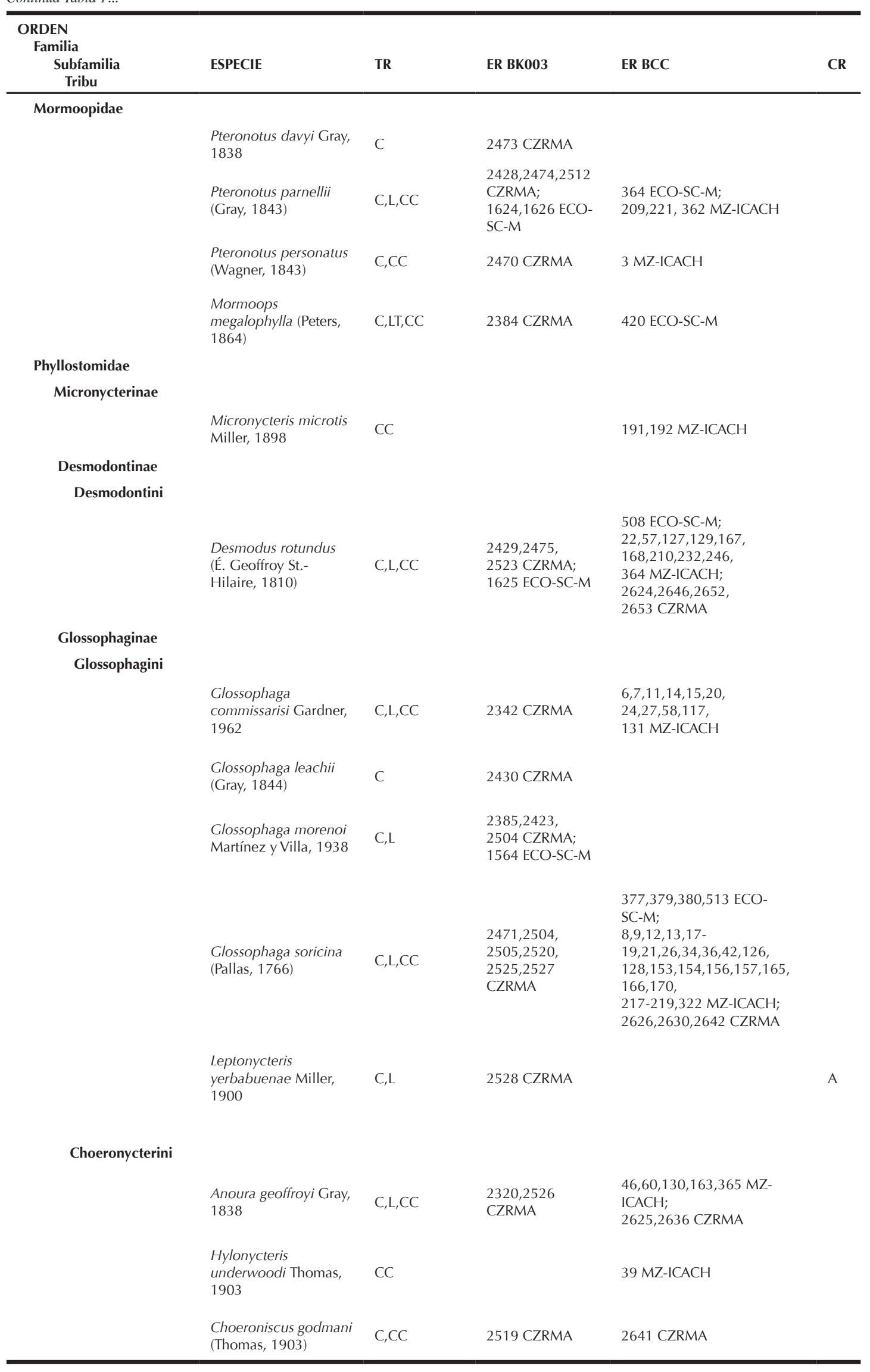


Continúa Tabla 1...

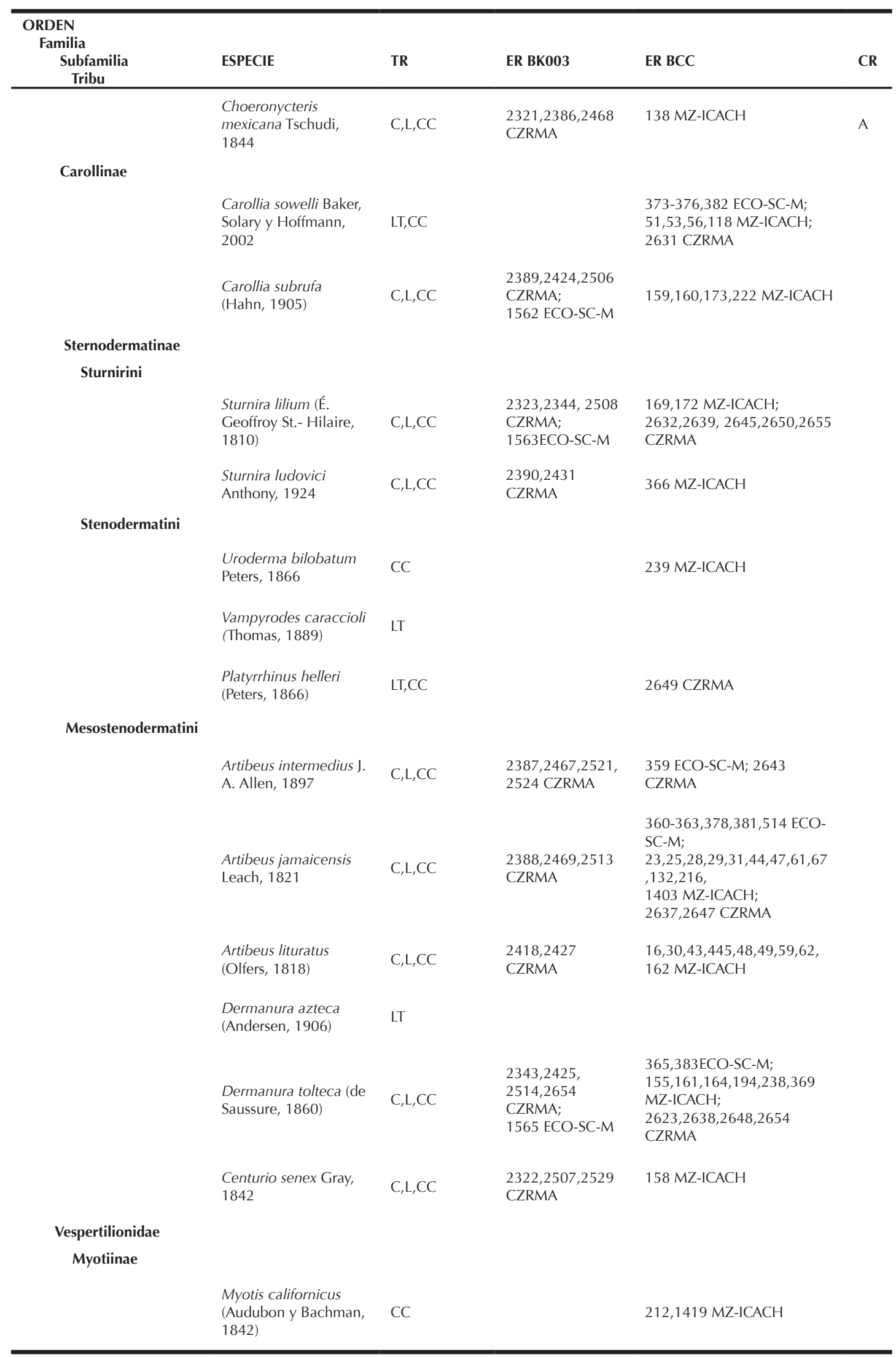




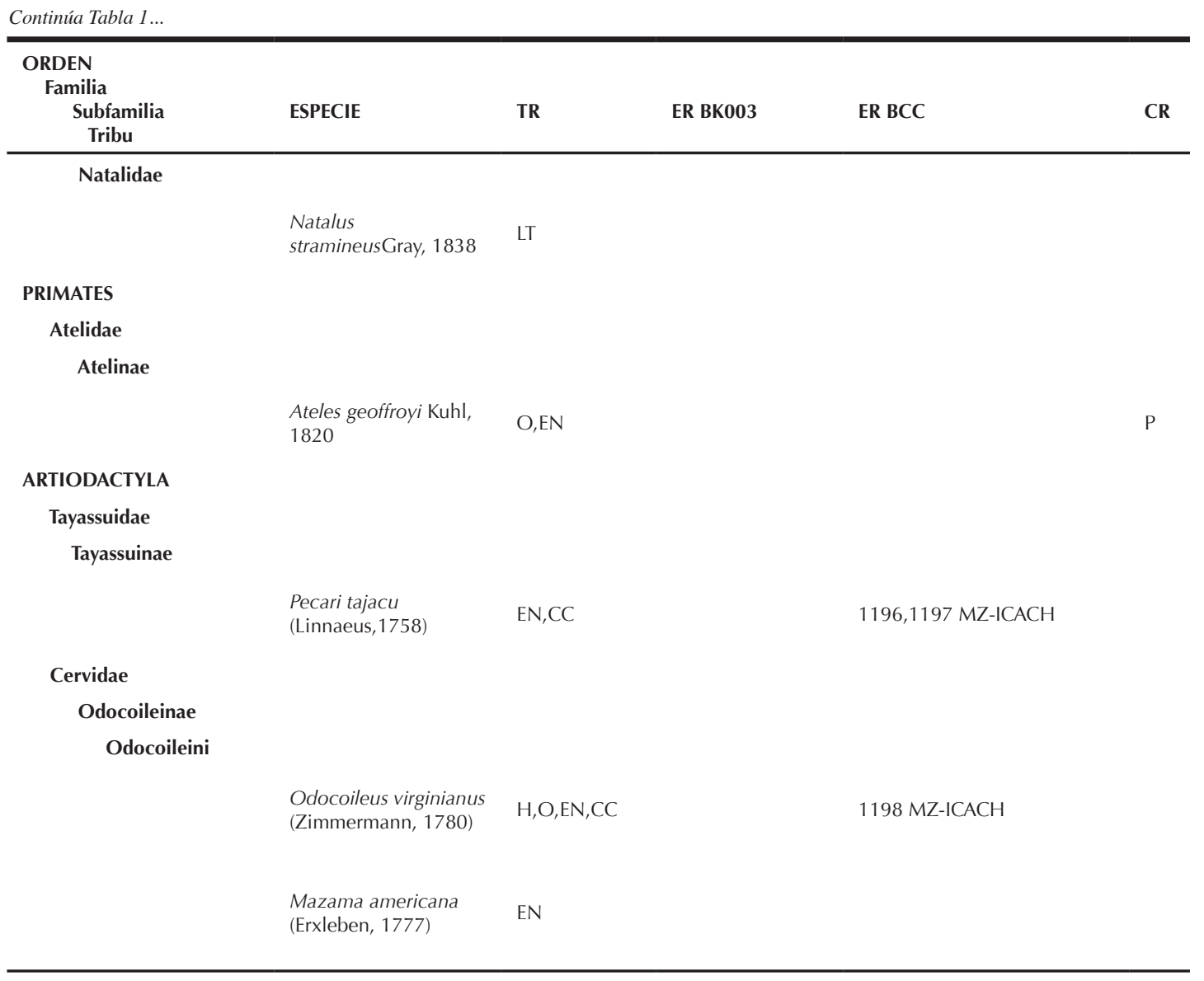

TR, tipo de registro como sigue: durante el proyecto BK003. C: colectado; L: liberado; O: observado; H: Huella; EX: excreta, EN: entrevista. Otras fuentes.- LT: literatura (Gálvez-Altúzar 1990; Riechers y De la Cruz 2012); CC: registro mediante consulta de bases de datos de Colecciones Científicas. ER BK003, Ejemplares recolectados durante el proyecto BK003 e incorporados a la CZRMA o ECO-SC-M. ER BCC, Ejemplare referidos en base de datos de colecciones científicas estatal y nacional. Se indica el número de catálogo del ejemplar depositado en la CZRMA o ECO-SC-M, y referido en la base de datos de colecciones científicas. Las abreviaturas de las colecciones son como sigue: Colección de Mamíferos de El Colegio de la Frontera Sur San Cristóbal de las Casas (ECO-SC-M); Museo de Zoología de la Universidad de Ciencias y Artes de Chiapas (MZ-ICACH); Colección Zoológica Regional Mammalia de la Secretaria del Medio Ambiente e Historia Natural (CZRMA). CR, categoría de riesgo de acuerdo con la NOM-059-SEMARNAT-2010: P, en Peligro de Extinción; A, Amenazada; Pr, Sujeta a Protección Especial.

El orden Chiroptera (40.26\%) fue el que presentó el mayor número de especies (31), seguido de los órdenes Rodentia (20 especies, 25.97\%) y Carnivora (14 especies, $18.8 \%$, Fig. 3). Lo anterior coincide con lo reportado por diversos autores (Ramírez-Pulido y Castro Campillo 1994; Retana y Lorenzo 2002), quienes citan a los dos primeros grupos como los principales componentes de la riqueza de especies de mamíferos en México y en Chiapas. Los siete órdenes restantes (Didelphimorphia, Cingulata, Pilosa, Lagomorpha, Soricomorpha, Primates y Artiodactyla) estuvieron representados, cada uno, por menos del $4 \%$ de las especies presentes en el área de estudio (Fig. 3).

La alta riqueza de murciélagos en la zona de estudio puede deberse a la amplia variedad de recursos alimentarios que explotan (frutas, polen, néctar, pequeños vertebrados, insectos, otros artrópodos y sangre); la diversidad de hábitats que ocupan, ya que en el Parque Cañón del Sumidero encontramos desde selvas bajas hasta bosques de encinos; su gran capacidad de desplazamiento en busca de refugio y alimento; así como la presencia de especies generalistas que pueden tolerar la modificación de los ambientes donde habitan (Cruz-Lara et al. 2004). 
Los roedores también presentaron una alta riqueza en el Parque (25.97\%), posiblemente debido a que comprenden una amplia gama de nichos ecológicos (LópezArévalo et al. 1993; López-Vidal y Álvarez 1993), explotando hábitats naturales como selvas y bosques, hasta áreas transformadas por el hombre como cultivos (maizal, cafetal, pastizales).

Los carnívoros son el tercer grupo más importante, ya que con sus 14 especies constituyen el $18.18 \%$ de la mastofauna del Parque. Es importante recalcar que en el área de estudio se obtuvo el registro de félidos pequeños (Herpailurus yagouaroundi, Leopardus pardalis y L. wiedii), pero no de carnívoros mayores como el puma (Puma concolor) y jaguar (Panthera onca), aunque dueños de cafetales refieren haber hecho avistamientos de jaguar en el Mirador El Roblar y a $2 \mathrm{~km}$ del Mirador La Atalaya durante la década de los 80's del siglo XX. Tal vez, no fue posible el registro de estas especies porque se distribuyen en áreas de difícil acceso; además, el jaguar se caracterizan por su alta sensibilidad a las perturbaciones humanas.

Los hábitats que presentaron mayor riqueza de especies fueron: la SM con el $33.8 \%$ de la mastofauna del Parque (26 especies), seguido del AP2 con 28.6\% (22 especies), AP3 y la SBC2 con el $27.3 \%$ cada uno (ambos con 21 especies) y la SBC1 con el 26\% (20 especies); los demás hábitats presentaron valores por debajo del 25\% (Tabla 2). Posiblemente, la SM presentó una alta riqueza porque es una de las áreas más conservadas del Parque, y por lo tanto, alberga especies de mamíferos silvestres sensibles o medianamente sensibles a la perturbación humana (e. g., Bassariscus sumichrasti, Choeronycteris mexicana y Tylomys nudicaudus), además de presentar el mayor número de especies exclusivas con respecto de los otros hábitas (e. g., Mustela frenata, Neotoma mexicana, Peromyscus aztecus, P. melanophrys y T. nudicaudus).

Por su parte, el AP2 y el AP3, tienen también una alta riqueza de mamíferos, esto podría explicarse porque ambos hábitats presentan gran heterogeneidad ambiental que incluye, una diversidad de cultivos (maíz, frutales, café y/o caña), parches de encinares (Quercus sp.), acahuales, pastizales inducidos (zacate estrella) y cuerpos de agua inducidos permanentemente (jagüeyes), arroyos naturales y cultivos de café en los alrededores, lo que proporciona refugio y diversidad en recursos alimenticios para la mastofauna silvestre. En la escala local las acciones humanas tienen efectos importantes en las comunidades de la vida silvestre, que pueden beneficiar a determinadas especies (Arellano y Halffter 2003). De hecho, en estos hábitats se registró a Desmodus rotundus, la cual se ve favorecida por las actividades ganaderas, así como Pteronotus parnellii, $P$. davyi, Sigmodon hispidus y Sylvilagus floridanus, las cuales aprovechan los pastizales. Esto coincide con las observaciones de Parmenter et al. (1995), quienes afirman que algunos disturbios antropogénicos incrementan la diversidad de especies debido al incremento espacial y temporal de la heterogeneidad del hábitat. Las especies exclusivas de estos hábitats perturbados incluyen dos murciélagos (Glossophaga leachii y Pteronotus davyi), dos roedores (Heteromys desmarestianus y Peromyscus levipes) y un carnívoro (Potos flavus). Las especies de murciélagos $G$. leachii y $P$. davyi posiblemente utilizaron estos hábitats perturbados como sitio de paso en busca de alimento o de descanso, ya que tienen gran capacidad de desplazamiento, hasta 100 km de distancia (Fleming 1993).

En el caso de los roedores Heteromys desmarestianus y Peromyscus levipes, son especies abundantes en ambientes naturales y perturbados, asociados a cafetales y zonas 
de cultivo (Castro-Arellano y Santos 2005), posiblemente se ven beneficiados porque los cultivos (maíz) están sometidos a pequeñas quemas que favorecen al renuevo del estrato herbáceo, incrementando el recurso alimenticio (Gashwiler 1959). Caso contrario para $P$. flavus, que prefiere sitios no perturbados y selvas, sin embargo se le ha reportado en selvas riparias y secundarias y ocasionalmente en huertos (Figueroa y Arita 2005). Las condiciones estructurales de las áreas perturbadas proporcionan diferentes estados de sucesión y números de estratos de vegetación, lo que aumenta que las posibilidades de obtención de alimento y refugio sean mayores que en otros hábitats, lo que aumenta la diversidad local (Rappole et al. 2003).

La alta riqueza de especies de la SBC1 y la SBC2 pueden deberse a que incluyó dos sitios que se encuentran al margen del Río Grijalva (Predio El Jardín y Rancho Magaly), que proporciona permanentemente a los mamíferos un recurso tan importante como es el agua. Además, al presentar una topografía muy accidentada y pedregosa, estos hábitats facilitan un gran número de madrigueras, sobre todo a pequeños roedores (e. g., Baiomys musculus, Liomys pictus, Oligorizomys fulvescens, Oryzomys couesi y Peromyscus mexicanus) y mamíferos medianos excavadores (Conepatus leuconotus y Dasypus novemcinctus). Asimismo, contar con una cubierta de hojarascas y materia vegetal en descomposición, permite la existencia de pequeños invertebrados (insectos, lombrices, miriápodos, arácnidos) que son fuente de alimento para especies omnívoras de mamíferos (e. g., C. leuconotus, D. novemcinctus, Nasua narica, Cuniculus paca y Didelphis marsupialis). En estos hábitats, específicamente en la SBC1, se registraron dos individuos de Ateles geoffroyi, pero se piensa que solo lo utilizaron como sitio de paso, ya que las poblaciones de estos primates se tienen registradas muy cerca de este sitio en la selva alta o mediana subperennifolia, al margen del río Grijalva. Lo que concuerda con Silva-López et al. (1987), mencionan que A. geoffroyi se les observa en los estratos de la selva, aunque cruzan zonas de vegetación secundaria.

El ENC presentó una baja riqueza de especies (19 especies), esto es similar a lo reportado por Vidal-López (1998), en otra área de estudio en Chiapas. La baja riqueza puede deberse a que estos sitios tienen una estructura vegetal menos compleja, en comparación con las selvas. Además, estos bosques son fuertemente explotados por los pobladores locales, quienes extraen leña y madera para fines domésticos. En este hábitat se presentaron sólo dos especies exclusivas Cryptotis mexicana y Choeroniscus godmani. La primera especie es exclusiva de hábitats húmedos, registrándose en México sólo en bosques húmedos de pino y bosque mesófilo (Castro-Arellano y Ceballos 2005), lo cual el encinar en el área de estudio cubre con esta características de humedad que requiere C. mexicana. Para el caso de C. godmani, se ha registrado en selvas tropicales caducifolias, pero también en bosques húmedos (Arita 2005a), posiblemente el registro de C. godmani en el encinar del Parque Nacional esté relacionada con la presencia de arbustos de Calliandra houstoniana, fuente potencial de alimento para especies de murciélagos nectarívoros (Riechers et al. 2003), considerando que C. godmani consume néctar y polen de diversas especies de flores (Gardner 1977).

En este estudio la SA presentó una menor riqueza de mamíferos (18 especies), posiblemente porque este hábitat solo ocupa una pequeña parte de la superficie en el área de estudio. La superficie reducida de SA dentro del paisaje, posiblemente esté siendo afectada por los cultivos circundantes, lo que puede originar la disminución de 
los valores de diversidad de las especies de mamíferos en este tipo de vegetación, ya que el proceso histórico que han sufrido los parches de vegetación conservados, al estar aislados, ha derivado en un empobrecimiento en la diversidad de las especies en el largo plazo (Sodhi et al. 2008). Este hábitat SA, presentó una especie indicadora de calidad de hábitat (Choeronycteris mexicana) con categoría de riesgo, de acuerdo a la Norma Oficial Mexicana (Diario Oficial de la Federación 2010), aún a pesar de presentar cultivos de café bajo, pero como se trata de una actividad agrícola que se caracteriza por ser orgánica de sombra, bajo un sistema de policultivo tradicional, permanece como uno de los hábitats mejor conservado en el Cañón del Sumidero. Existen evidencias de que algunos mamíferos voladores, como los quirópteros frugívoros, se han visto favorecidos por los árboles y arbustos presentes en y alrededor de las plantaciones de café de sombra (Williams-Guillén y Perfecto 2010, García-Estrada et al. 2012).

Cabe mencionar que Ateles geoffoyi y Tamandua mexicana, también especies con categoría de riesgo, se registraron solo en selva alta o mediana subperennifolia, pero en otros sitios que se encuentran al margen del río Grijalva (las cuales no fueron estudiadas), estas especies fueron registradas durante los recorridos en el río por lancha.

Figura 3. Composición taxonómica de mamíferos en el Parque Nacional Cañón del Sumidero, Chiapas.

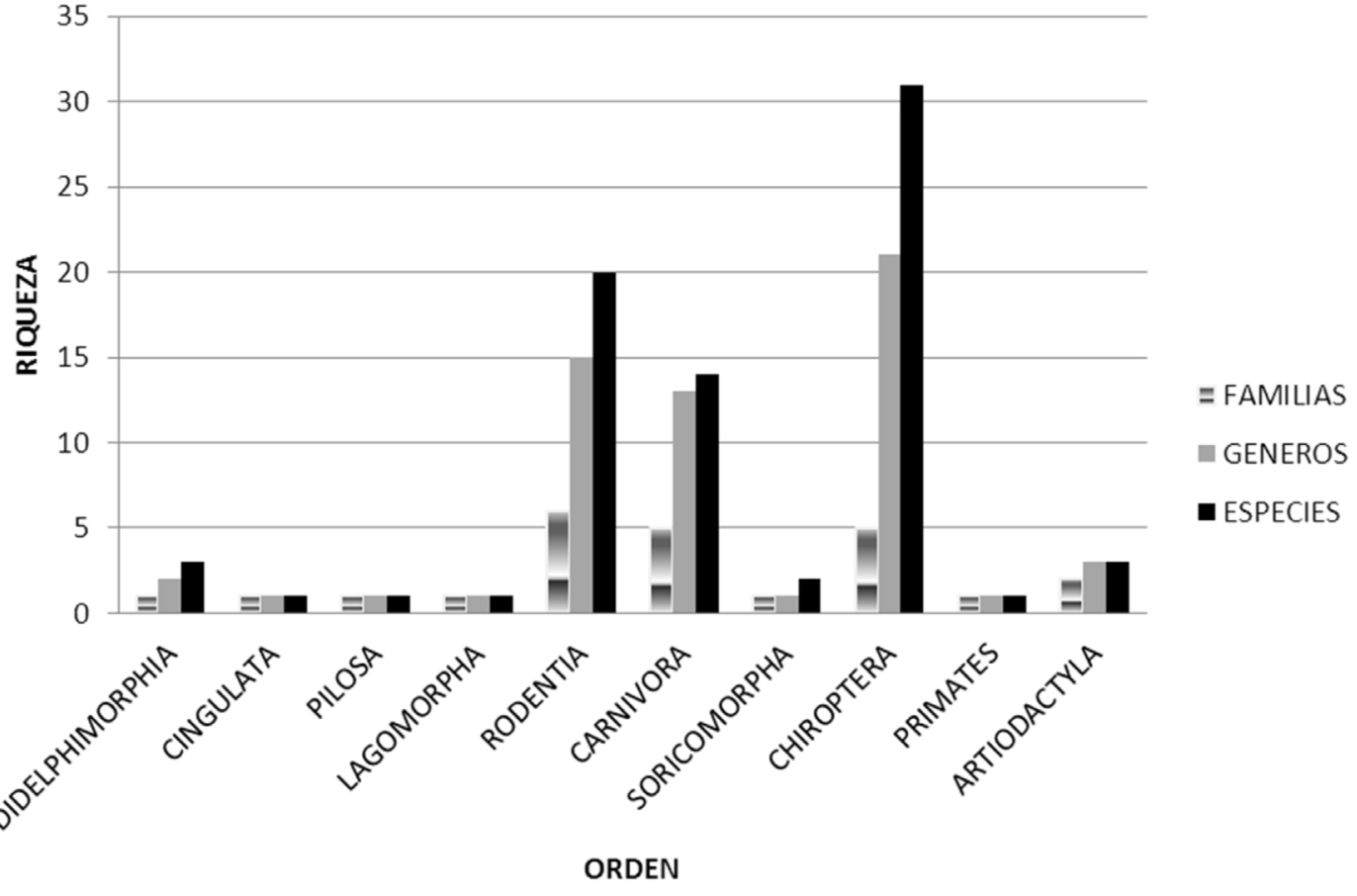

Abundancia relativa. Las especies con mayor abundancia relativa en el área de estudio fueron Artibeus jamaicensis (24.45\%), seguido de Peromyscus mexicanus (13.6\%), Sturnira lilium (9.19\%) y Liomys pictus $(6.53 \%)$. Las especies restantes presentaron valores por debajo del 6\% (Tabla 2). La abundancia de las cuatro especies mencionadas podría deberse a sus hábitos generalistas, por lo que se ven beneficiadas por la perturbación, adaptándose fácilmente a los diferentes nichos ecológicos disponibles, lo cual les confiere una ventaja competitiva frente a las demás especies (Cameron y Spencer 1981; Harris y Woolard 1990; Horváth et al. 2001; Marby et al. 2003).

Estas especies (A. jamaicensis, P. mexicanus, S. lilium y L. pictu) se registraron en los ocho hábitats muestreados del Parque Nacional. Además, con la excepción de L. pictus, 
se observó que estas mismas especies fueron muy abundantes en áreas perturbadas, incluyendo diversos cultivos (maíz, pastizal, frutales y café), las cuales constituyen hábitats muy dinámicos que les proporcionan refugio y alimento.

Por el contrario, 13 especies fueron poco abundantes con valores de 0.09\% cada una, ya que son especies sensibles a la perturbación y requieren de hábitat en buen estado de conservación o con poca perturbación como Tamandua mexicana (Cuarón 2005), Peromyscus melanophrys (Aragón 2005) y Tylomys nudicaudus (Espinoza-Medinilla 2005); o son especies que son raras por naturaleza, tanto por su área de distribución restringida como por su baja abundancia local como Choeroniscus godmani (Arita 1993) y Glossophaga leachii (Arita 2005b); o son especies que requieren características específicas en su hábitat como suelos rocosos y sotobosques densos con abundancia de humus como Cryptotis mexicana (Vargas et al. 2009) y Peromyscus aztecus (RamírezPulido et al. 2005a); o especies que si bien han sido registradas por diversos autores como abundantes con tolerancias a las perturbaciones antrópicas, su baja representatividad en el presente estudio probablemente se debió a que los registros fueron ocasionales, ya que se desplazan en alturas elevadas en busca de insectos para su alimento, lo que hace difícil su captura como Mormoops megallphylla, Pteronotus personatus y Pteronotus davyi (Nebel y Wrigth 1999), o bien, porque debido a su tamaño pequeño o mediano no son tan visibles y a eso se suma que tienen hábitos crepusculares o nocturnos como Spilogale gracilis (Ceballos 2005), Mustela frenata (Ceballos y Oliva 2005) y Neotoma mexicana (Zarza y Ceballos 2005).

Categoría de riesgo. De las 77 especies presentes en el Parque Nacional, el 14.3\% (11 especies; Tabla 1) se encuentran en alguna categoría de riesgo, de acuerdo con la Norma Oficial Mexicana NOM-059-SEMARNAT-2010 (Diario Oficial de la Federación 2010). De éstas, cinco se encuentran en peligro de extinción (Ateles geoffroyi, Eira barbara, Leopardus wiedii, L. pardalis y Tamandua mexicana), cuatro están amenazadas (Coendou mexicanus, Choeronycteris mexicana, Herpailurus yagouaroundi y Leptonycteris curasoae) y dos están sujetas a protección especial (Bassariscus sumichrasti y Potos flavus). La mayoría de estas especies corresponden a mamíferos medianos y grandes con ámbitos hogareños relativamente extensos, por lo que son más vulnerables a la fragmentación del hábitat (Emmons y Feer 1997; Reid 1997). Para el caso del único primate $(A$. geoffoyi), el estado de conservación en el Parque se agrava porque su distribución fue muy localizada, registrándose en un fragmento de selva alta o mediana al margen del río Grijalva, además de registrar una baja abundancia relativa (1.47\%).

En el caso de mamíferos pequeños como los murciélagos, la destrucción de los refugios diurnos y sitios de crianza se encuentran entre las principales causas de declinación en sus poblaciones (Naranjo et al. 2005). En el área de estudio se registraron dos especies de murciélagos amenazados (C. mexicana y L. curasoae), en hábitats que podían cubrir sus requerimientos especiales con asociaciones de especies vegetales que les proporcionan alimento (Musa sp, Calliandra sp. y Agave grijalvensis), por lo que su estado de conservación se hace más vulnerable, además de que son poco abundantes en el área de estudio.

Para $L$. yerbabuenae se tiene documentada una colonia migratoria de maternidad entre octubre a enero en la cueva denominada Los Laguitos ubicada al norte de la ciudad 
de Tuxtla Gutiérrez, esta cueva está rodeada por vegetación de selva baja caducifolia (Riechers et al. 2003). Este murciélago nectarívoro podría estar utilizando el AP1 como zona de paso en busca de alimento, ya que solo se registraron dos individuos en este hábitat durante septiembre de 2005, y el hábitat muestreado presentaba cultivos de maíz con árboles frutales como plátano (Musa sp.), especie vegetal que ha sido documentado como fuente de alimento para L. yerbabuenae (Villa 1966).

Asimismo, para C. mexicana se registraron cuatro individuos en tres hábitats, SM, SA y SBC2, siendo los dos primeros las áreas mejores conservadas del parque, lo que apoya el criterio de Riechers y Vidal (2009) para considerarla como una especie sensible a las modificaciones antropogénicas. Si bien el hábitat SBC2 no es un área conservada, presenta árboles frutales como plátano (Musa sp.) posible fuente de alimento para $C$. mexicana.

Tabla 2. Porcentaje de abundancia relativa (\%AR), riqueza de especies y diversidad alfa de mamíferos en el Parque Nacional Cañón del Sumidero, Chiapas, de acuerdo con su presencia en los hábitats muestreados durante junio del 2004 a noviembre del 2005.

\begin{tabular}{|c|c|c|c|c|c|c|c|c|c|}
\hline \multirow{2}{*}{ ESPECIE } & \multirow{2}{*}{$\mathrm{AR} \%$} & \multicolumn{8}{|c|}{ HÁBITAT MUESTREADOS } \\
\hline & & ENC & SA & SM & SBC1 & SBC2 & AP1 & AP2 & AP3 \\
\hline Artibeus jamaicensis & 24.45 & $*$ & $*$ & $*$ & $*$ & * & $*$ & $*$ & $*$ \\
\hline Peromyscus mexicanus & 13.60 & $*$ & $*$ & $*$ & $*$ & $*$ & $*$ & $*$ & $*$ \\
\hline Sturnira lilium & 9.19 & $*$ & $*$ & $*$ & $*$ & * & $*$ & $*$ & $*$ \\
\hline Liomys pictus & 6.53 & * & $*$ & $*$ & $*$ & * & * & * & * \\
\hline Glossophaga soricina & 5.61 & * & & & $*$ & * & $*$ & & $*$ \\
\hline Dermanura tolteca & 4.23 & $*$ & $*$ & $*$ & $*$ & $*$ & $*$ & $*$ & $*$ \\
\hline Carollia subrufa & 3.95 & & & & $*$ & $*$ & & $*$ & $*$ \\
\hline Sciurus aureogaster & 3.40 & $*$ & * & $*$ & $*$ & $*$ & & $*$ & $*$ \\
\hline Urocyon cinereoargenteus & 3.40 & $*$ & $*$ & $*$ & $*$ & $*$ & & & $*$ \\
\hline Artibeus intermedius & 2.67 & $*$ & $*$ & $*$ & $*$ & * & $*$ & $*$ & $*$ \\
\hline Baiomys musculus & 2.11 & $*$ & $*$ & & $*$ & & & & $*$ \\
\hline Sigmodon hispidus & 1.93 & & $*$ & $*$ & & & $*$ & & $*$ \\
\hline Ateles geoffroyi & 1.47 & & & & $*$ & & & & \\
\hline Anoura geoffroyi & 1.38 & & $*$ & $*$ & & & $*$ & $*$ & \\
\hline Glossophaga morenoi & 1.29 & $*$ & $*$ & & $*$ & $*$ & $*$ & & $*$ \\
\hline Dasypus novemcinctus & 1.19 & $*$ & $*$ & $*$ & & $*$ & & & \\
\hline Orthogeomys grandis & 1.10 & $*$ & & & & $*$ & & & \\
\hline Nasua narica & 1.01 & & & $*$ & & $*$ & & $*$ & \\
\hline Sturnira ludovici & 1.01 & $*$ & $*$ & & $*$ & $*$ & & * & \\
\hline Glossophaga commissarisi & 0.83 & & & $*$ & & & * & & \\
\hline Pteronotus parnellii & 0.83 & & & & $*$ & & & $*$ & $*$ \\
\hline Desmodus rotundus & 0.74 & & & & $*$ & & $*$ & $*$ & $*$ \\
\hline Artibeus lituratus & 0.64 & & & & & & $*$ & & $*$ \\
\hline Conepatus leuconotus & 0.64 & $*$ & $*$ & & $*$ & $*$ & & & \\
\hline Odocoileus virginianus & 0.64 & $*$ & & $*$ & & & & & \\
\hline Sylvilagus floridanus & 0.64 & $*$ & & $*$ & & & * & & * \\
\hline Centurio senex & 0.55 & & $*$ & $*$ & & & $*$ & $*$ & $*$ \\
\hline
\end{tabular}


Continúa Tabla $2 \ldots$

\begin{tabular}{|c|c|c|c|c|c|c|c|c|c|}
\hline Peromyscus levipes & 0.46 & & & & & & & $*$ & \\
\hline Bassariscus sumichrasti & 0.37 & & & $*$ & & & & $*$ & \\
\hline Cuniculus paca & 0.37 & & & & & $*$ & & & \\
\hline Choeronycteris mexicana & 0.37 & & $*$ & $*$ & & $*$ & & & \\
\hline Oligorizomys fulvescens & 0.37 & & & & $*$ & & & $*$ & $*$ \\
\hline Heteromys desmarestianus & 0.28 & & & & & & & $*$ & \\
\hline Oryzomys couesi & 0.28 & & & & $*$ & & & $*$ & $*$ \\
\hline Reithrodontomys gracilis & 0.28 & & $*$ & & & & & & \\
\hline Didelphis marsupialis & 0.18 & & & & & $*$ & & & \\
\hline Didelphis virginiana & 0.18 & & & $*$ & * & & & & \\
\hline Leptonycteris curasoe & 0.18 & & & & & & * & & \\
\hline Potos flavus & 0.18 & & & & & & & $*$ & \\
\hline Procyon lotor & 0.18 & & & & & & & $*$ & \\
\hline Cryptotis mexicana & 0.09 & $*$ & & & & & & & \\
\hline Choeroniscus godmani & 0.09 & $*$ & & & & & & & \\
\hline Glossophaga leachii & 0.09 & & & & & & & $*$ & \\
\hline Mormoops megallophylla & 0.09 & & & & & $*$ & & & \\
\hline Mustela frenata & 0.09 & & & $*$ & & & & & \\
\hline Neotoma mexicana & 0.09 & & & $*$ & & & & & \\
\hline Peromyscus aztecus & 0.09 & & & $*$ & & & & & \\
\hline Peromyscus melanoprhys & 0.09 & & & * & & & & & \\
\hline Pteronotus personatus & 0.09 & & & & & $*$ & & & \\
\hline Pteronotus dayvi & 0.09 & & & & & & & & $*$ \\
\hline Spilogale gracilis & 0.09 & & & $*$ & & & & & \\
\hline Tamandua mexicana & 0.09 & & & & & & & & \\
\hline Tylomys nudicaudus & 0.09 & & & $*$ & & & & & \\
\hline Abundancia relativa total & 100.00 & & & & & & & & \\
\hline Riqueza de especies & & 19 & 18 & 26 & 20 & 21 & 16 & 22 & 21 \\
\hline $\begin{array}{l}\text { Diversidad alfa } \mathbf{H}^{\prime} \text { (Shannon- } \\
\text { Wiener) }\end{array}$ & & 2.51 & 2.07 & 2.52 & 1.73 & 2.37 & 2.25 & 2.29 & 2.13 \\
\hline
\end{tabular}

Abreviatura para el hábitat: ENC, encinar; SA, selva alta o mediana subperennifolia con cafetales de sombra; SM, selva mediana o baja perennifolia; SBC1, selva baja caducifolia; SBC2, selva baja caducifolia con cultivos (frijol y maíz) y árboles frutales; AP1, área perturbada con selva alta o mediana subperennifolia, potreros, acahuales y cultivo de maíz; AP2, área perturbada con pinos, cafetales de sombra y potreros; AP3, área perturbada con encinares, con selva alta o mediana subperennifolia y pastizal de zacate estrella.

De acuerdo a Primack (1998), la fragmentación es ocasionada por la apertura de nuevas áreas de pastoreo y cultivos, así como por la expansión de centros de población humana, lo que puede provocar aislamiento reproductivo entre las poblaciones de una especie y entrecruzamiento, por parte de los individuos de la población aislada, con la subsecuente pérdida de variación genética. Para el caso del Parque Nacional Cañón del Sumidero, desafortunadamente también se observó que el hábitat original se ha modificado debido a los nuevos asentamientos irregulares de población humana (colonia Emiliano Zapata 
y Loma Larga en el municipio de Tuxtla Gutiérrez) en los límites del polígono del área natural, así como por el crecimiento de la frontera agrícola y la apertura de vías terrestres y fluviales (Malecón de Chiapa de Corzo).

Diversidad alfa. Mediante el índice de Shannon-Wiener $\left(\mathrm{H}^{\prime}\right)$ se encontró que el hábitat con mayor diversidad alfa fue la SM (2.52), seguido del ENC (2.51), de la SBC2 (2.37) y el AP2 (2.29, Tabla 2), aunque no se detectaron diferencias estadísticamente significativas entre ellos (Tabla 3). Estos hábitats más diversos presentaron una riqueza desde 19 a 26 especies con un total de individuos de 87 a 149, pero con uniformidad en los registros de cada especies, siendo el máximo 36 individuos en cada una de las siguientes especies: Artibeus jamaicensis, Peromyscus mexicanus, Sturnira lilium, Glossopaga soricina y/o Liomys pictus. El ENC presentó una baja riqueza de especies (19), con una alta diversidad alfa (2.51), ya que el número de individuos de las especies fueron más uniformes.

La SBC1 presentó un valor significativamente más bajo en diversidad alfa (1.73), con respecto de los restantes siete hábitats (Tabla 2, Tabla 3). Este hábitat alcanzó una riqueza de 20 especies de mamíferos con un total de 230 individuos, pero con dominancia de $A$. jamaicensis $(n=124)$, lo que repercutió en la baja diversidad alfa. En Dzilam de Bravo, Yucatán, Cimé-Pool et al. (2010) encontraron que existe mayor diversidad de roedores en sitios perturbados como los pastizales con respecto a las selvas bajas caducifolias espinosas con diferente grado de recuperación. Asimismo Sosa et al. (2008) reportan en la zona montañosa de Veracruz, que la menor diversidad de murciélagos fue en un fragmento de bosque mesófilo de montaña en relación con ocho fincas cafetaleras con diferente manejo, atribuyendo esta baja diversidad a la dominancia de Sturnira ludovici que aportó el $40 \%$ de las capturas. Caso contrario refieren García-Estrada et al. (2006), donde la mayor diversidad de murciélagos se encontró en el bosque mesófilo de montaña con relación a cinco sitios con plantaciones de café con diferente grado de manejo en Motozintla y Tapachula, Chiapas.

Otros autores señalan que no hallaron discrepancias en la diversidad de mamíferos por la modificación del ambiente, como es el caso de García-Estrada et al. (2002), que no encontraron diferencias en la diversidad de especies de pequeños roedores por la alteración del hábitat en selvas bajas caducifolias en el sureste de Morelos. Asimismo, Cruz-Lara et al. (2004), mencionan que la diversidad de especies de mamíferos en cafetales con sombra fue similar a la registrada en selva mediana subperennifolia en la comunidad de Loma Bonita, en Maravilla Tenejapa, Chiapas.

Según Fjeldsa et al. (2012), refieren que a escala local los cambios en la heterogeneidad pueden ser la principal causa de las diferencias en la diversidad alfa, donde ocurre la agregación de muchas especies a pequeñas distancias, como en el caso de las zonas de montañas.

Diversidad beta de la mastofauna. La diversidad beta entre hábitats fue intermedia (desde 0.22 hasta 0.58), con los valores más altos entre el AP 1 y el AP2 (0.58), seguido de la SM con la SBC1 y del AP1 con la SBC2 (0.57 cada uno; Tabla 4). El valor más bajo se presentó entre la SBC1 con el AP3 (0.22), seguido del ENC con la SM, del ENC con la SBC2, y del AP1 con el AP2 (0.30 cada uno, Tabla 4). Por lo tanto, la mayor similitud (Cs 
0.78) se encontró en la SBC1 con el AP3, ya que compartieron 16 de las 20 y 21 especies que presentan, respectivamente (Tabla 4), posiblemente se debe a que las especies que comparten está dada por murciélagos frugívoros que son de amplia distribución y hábitos generalistas (e. g., Artibeus jamaicensis, A. intermedius, Dermanura tolteca, Sturnira lilium), y pequeños roedores que no son tan sensibles a la perturbación del hábitat (e. g., Liomys pictus, Baiomys musculus, Oligorizomys fulvescens, Oryzomys couesi).

Los hábitats muestreados presentaron una diversidad beta intermedia, por lo tanto las especies de mamíferos entre hábitat tienen una importante complementariedad, lo que contribuye a que el Parque Nacional Cañón del Sumidero albergue un importante riqueza de especies de mamíferos (77). Está área natural protegida presenta un paisaje heterogéneo que va desde cultivos de maíz, frutales y cafetales, acahuales, pastizales inducidos, además de diferencias en el gradiente altitudinal desde los 390 a los 1,258 msnm, por lo tanto se presenta diversidad en los tipos de vegetación desde selvas bajas caducifolias, selvas medianas o bajas perennifolias, selvas altas o medianas subperennifolias hasta encinares.

\begin{tabular}{|c|c|c|c|c|c|c|c|}
\hline HÁBITAT & SA & SM & SBC1 & SBC2 & AP1 & AP2 & AP3 \\
\hline ENC & $\begin{array}{l}t=3.303 \\
g l=33.14\end{array}$ & $\begin{array}{l}t=0.057 \\
\mathrm{gl}=44.958\end{array}$ & $\begin{array}{l}t=6.376 \\
\mathrm{gl}=38.02\end{array}$ & $\begin{array}{l}t=1.182 \\
g l=39.49\end{array}$ & $\begin{array}{l}t=1.835 ; \\
\mathrm{g} \mid=28.3\end{array}$ & $\begin{array}{l}t=1.312 ; \\
\mathrm{g} \mid=33.144\end{array}$ & $\begin{array}{l}t=2.718 ; \\
\mathrm{gl}=36.275\end{array}$ \\
\hline SA & & $\begin{array}{l}t=3.113 \\
\mathrm{gl}=39.978\end{array}$ & $\begin{array}{l}t=2.371 ; \\
\mathrm{gl}=36.698\end{array}$ & $\begin{array}{l}t=2.096 \\
g \mid=36.958\end{array}$ & $\begin{array}{l}t=1.113 ; \\
\mathrm{g} \mid=33.187\end{array}$ & $\begin{array}{l}t=1.219 ; \\
\mathrm{g} \mid=38.491\end{array}$ & $\begin{array}{l}t=0.394 ; \\
\mathrm{gl}=38.992\end{array}$ \\
\hline SM & & & $\begin{array}{l}t=5.89 ; \\
g \mid=45.287\end{array}$ & $\begin{array}{l}t=1.133 ; \\
g \mid=46.724\end{array}$ & $\begin{array}{l}t=1.763 ; \\
\mathrm{g} \mid=34.235\end{array}$ & $\begin{array}{l}t=1.289 ; \\
\mathrm{g}=38.521\end{array}$ & $\begin{array}{l}t=2.582 ; \\
g \mid=43.122\end{array}$ \\
\hline SBC1 & & & & $\begin{array}{l}t=4.839 ; \\
g \mid=40.889\end{array}$ & $\begin{array}{l}t=3.416 \\
\mathrm{gl}=32.206\end{array}$ & $\begin{array}{l}t=3.223 \\
\mathrm{gl}=36.973\end{array}$ & $\begin{array}{l}t=2.704 ; \\
\mathrm{gl}=39.813\end{array}$ \\
\hline SBC2 & & & & & $\begin{array}{l}t=0.788 ; \\
\mathrm{g}=32.115\end{array}$ & $\begin{array}{l}t=0.435 \\
\mathrm{~g}=36.778\end{array}$ & $\begin{array}{l}t=1.596 ; \\
\mathrm{g}=40.086\end{array}$ \\
\hline AP1 & & & & & & $\begin{array}{l}t=0.228 ; \\
\mathrm{gl}=37.911\end{array}$ & $\begin{array}{l}t=0.704 ; \\
g \mid=35.949\end{array}$ \\
\hline AP2 & & & & & & & $\begin{array}{l}t=0.857 \\
g l=41.1\end{array}$ \\
\hline
\end{tabular}

$t=\mathrm{t}$ de student modificada por Hutchenson, con probabilidad 0.05, gl=grados de libertad.

Abreviatura para el hábitat: ENC, encinar; SA, selva alta o mediana subperennifolia con cafetales de sombra; SM, selva mediana o baja perennifolia; SBC1, selva baja caducifolia; SBC2, selva baja caducifolia con cultivos (frijol y maíz) y árboles frutales; AP1, área perturbada con selva alta o mediana subperennifolia, potreros, acahuales y cultivos de maíz; AP2, área perturbada con pinos, cafetales y potreros; AP3, área perturbada con encinares, con selva alta o mediana subperennifolia y pastizal de zacate estrella.

Estudios similares reportan Gallina et al. (2008) y Sosa et al (2008), estos autores señalan que las especies de mamíferos tienen una importante complementariedad entre fincas cafetaleras y un fragmento de bosque mesófilo de montaña en Veracruz. Sosa et al. (2008), señalan que para la conservación de las comunidades de murciélagos es necesario mantener el paisaje heterogéneo con manchones de bosque, cafetales y otros agroecosistemas. Por su parte Gallina et al. (2008), mencionan que es importante mantener los agroecosistemas cafetaleros para proteger la mayor parte de mamíferos, pero el tipo de manejo de los cafetales ejerce un efecto negativo para mamíferos 
medianos, así como en la calidad del paisaje.

En cambio García-Estrada et al. (2006), refieren valores bajos de diversidad beta en especies de murciélagos en bosque mesófilo y plantaciones de café en el sureste de Chiapas, por lo tanto las especies de presentan una alta similitud; siendo las especies compartidas murciélagos frugívoros con distribución amplia y hábitos generalistas lo que facilita su desplazamiento entre la vegetación original, los cafetales y parches de vegetación secundaria en diferentes etapas de regeneración, aprovechando los diferentes refugios disponibles en la zona.

\begin{tabular}{|c|c|c|c|c|c|c|c|c|}
\hline \multirow{8}{*}{$\begin{array}{l}\text { Tabla 4. Comparación } \\
\text { de la diversidad beta de } \\
\text { la mastofauna entre los } \\
\text { hábitats muestreados, } \\
\text { mediante el inverso de } \\
\text { Similitud de Sorensen } \\
\text { cualitativo. }\end{array}$} & HÁBITAT & SA & SM & SBC1 & SBC2 & AP1 & AP2 & AP3 \\
\hline & ENC & $0.30(0.70) \underline{13}$ & $0.47(0.53) \underline{12}$ & $0.33(0.67) \underline{13}$ & $0.30(0.70) \underline{14}$ & $0.49(0.51) \underline{9}$ & $0.56(0.44) \underline{9}$ & $0.40(0.60) \underline{12}$ \\
\hline & SA & & $0.36(0.64) \underline{14}$ & $0.37(0.63) \underline{12}$ & $0.33(0.67) \underline{13}$ & $0.41(0.59) \underline{10}$ & $0.50(0.50) \underline{10}$ & $0.38(0.62) \underline{12}$ \\
\hline & SM & & & $0.57(0.43) \underline{10}$ & $0.49(0.51) \underline{12}$ & $0.48(0.52) \underline{11}$ & $0.54(0.46) \underline{11}$ & $0.53(0.47) \underline{11}$ \\
\hline & SBC1 & & & & $0.37(0.63) \underline{13}$ & $0.50(0.50) \underline{9}$ & $0.38(0.62) \underline{13}$ & $0.22(0.78) \underline{16}$ \\
\hline & SBC2 & & & & & $0.57(0.43) \underline{8}$ & $0.49(0.51) \underline{11}$ & $0.48(0.52) \underline{11}$ \\
\hline & AP1 & & & & & & $0.58(0.42) \underline{8}$ & $0.30(0.70) \underline{13}$ \\
\hline & AP2 & & & & & & & $0.44(0.56) \underline{12}$ \\
\hline
\end{tabular}

valores en cursiva, diversidad beta; ( valores entre paréntesis), Similitud de Sorensen Cualitativo; valores subrayados, especies compartidas

Abreviatura para el hábitat: ENC, encinar; SA, selva alta o mediana subperennifolia con cafetales de sombra; SM, selva mediana o baja perennifolia; SBC1, selva baja caducifolia; SBC2, selva baja caducifolia con cultivos (frijol y maíz) y árboles frutales; AP1, área perturbada con selva alta o mediana subperennifolia, potreros, acahuales y cultivo de maíz; AP2, área perturbada con pinos, cafetales y potreros; AP3, área perturbada con encinares, con selva alta o mediana subperennifolia y pastizal de zacate estrella.

\section{Conclusiones}

El Parque Nacional Cañón del Sumidero presenta una importante riqueza de mamíferos silvestres, que correspondiente al $37.5 \%$ de la mastofauna de Chiapas; siendo los murciélagos y los roedores los que presentaron la mayor riqueza de especies, en relación a los ocho órdenes restantes que se distribuyen en el parque. Esta área cobra mayor importancia ecológica por encontrarse adyacente a la ciudad de Tuxtla Gutiérrez, ya que además proporciona servicios ecológicos, mediante especies de mamíferos silvestres dispersoras de semillas, polinizadoras y controladoras de plagas.

La selva mediana o baja perennifolia es el hábitat más conservado respecto a los siete hábitats muestreados con diferente grado de perturbación, además presentó la mayor riqueza de especies de mamíferos y la mayor diversidad alfa; también alberga especies en alguna categoría de riesgo de acuerdo a la Norma Oficial Mexicana (Bassariscus sumichrasti, Choeronycteris mexicana) o especies sensibles a la modificación del hábitat, por lo tanto indicadoras de calidad de hábitat (Neotoma mexicana, Tylomys nudicaudus, Odocoileus virginianus). Las áreas perturbadas también presentaron una importante riqueza de especies de mamíferos, pero estas por lo general fueron especies comunes y tolerantes a ambientes modificados como Artibeus jamaicensis, Sturnira lilium, Peromyscus mexicanus y Liomys pictus, con las mayores abundancias relativas.

Lo anterior hace evidente que es necesario poner mayor atención en la conservación de las selvas medianas o bajas perennifolias, ya que son un refugio importante de la 
mastofauna, sobre todo de especies sensibles a la perturbación.

El parque presenta una heterogeneidad de paisaje que permite que exista una importante complementariedad de especies de mamíferos entre hábitats muestreados, con datos intermedios en la diversidad beta. El parque juega un papel importante como refugio de la biodiversidad, ya que de las 77 especies de mamíferos silvestres que alberga, alrededor del 15\% se encuentra en alguna categoría de riesgo, de acuerdo a la Norma Oficial Mexicana, por lo que es necesario establecer programas de conservación y manejo de las especies de mamíferos en las que se difundan la importancia ecológica y social, así como el valor escénico y ecológico del área, además de involucrar a los pobladores locales de las comunidades rurales para su implementación.

Nuestro inmenso agradecimiento a tres revisores anónimos que con sus comentarios y observaciones enriquecieron sustancialmente este escrito. A la Comisión Nacional para el Conocimiento y Uso de la biodiversidad por financiar el proyecto BK003, del cual deriva este artículo; a M. A. Altamirano G. O. responsable de dicho proyecto y a sus aportaciones a este manuscrito, a R. Vidal por su apoyo en campo. A los manejadores del Parque Nacional Cañón del Sumidero de la Comisión Nacional de Áreas Naturales Protegidas y a los pobladores locales, por las facilidades prestadas para realizar el trabajo de campo. A los responsables y técnicos de las colecciones mastozoológicas consultadas (MZ-ICACH, ECO-SC-M y CZRMA).

Altamirano-González, O. M. A., J. Guzmán, R. Luna, A. Riechers, y R. Vidal. 2007. Vertebrados Terrestres del Parque Nacional Cañón del Sumidero, Chiapas, México. Instituto de Historia Natural y Ecología. Informe Final. Sistema Nacional de Información sobre Biodiversidad-Comisión Nacional para el Conocimiento y Uso de la Biodiversidad. Proyecto No. BK003. Ciudad de México, México.

Álvarez, T., S. T. Álvarez-Castañeda, y J. C. López-Vidal. 1994. Claves para Murciélagos Mexicanos. Centro de Investigaciones Biológicas del Noroeste S. C.-Escuela Nacional de Ciencias Biológicas, Instituto Politécnico Nacional. La Paz, México.

Anthony, E. L. P. 1988. Age determination in bats. Pp. 47-58 in Ecological and Behavioral Methods for the Study of Bats (Kunz T. H., ed.). Smithsonian Institute. Washington, EE.UU.

Aragón, E. 2005. Peromyscus melanophrys (Coues, 1874). Pp. 753-754 en Los mamíferos silvestres de México (Ceballos, G., y G. Oliva, coords.). Comisión Nacional para el Conocimiento y Uso de la Biodiversidad-Fondo de Cultura Económica. Ciudad de México, México.

Aranda, M. 2000. Huellas y otros rastros de los mamíferos grandes y medianos de México. Comisión Nacional para el Conocimiento y Uso de la BiodiversidadInstituto de Ecología, A. C. Xalapa, México.

Aranda, M. J., E I. March. 1987. Guía de los mamíferos silvestres de Chiapas. Instituto Nacional de Investigaciones sobre Recursos Bióticos. Xalapa, México. 
Arellano, L., ANd G. Halffter. 2003. Gamma diversity: derived from and a determinant of alpha diversity and beta diversity. An analysis of three tropical landscapes. Acta Zoológica Mexicana (n. s.) 90:27-76.

Arita, H. T. 1993. Conservation biology of the cave bats of Mexico. Journal of Mammalogy 74:693-702.

Arita, H. T. 2005a. Choeroniscus godmani (Thomas, 1903). Pp. 211 en Los mamíferos silvestres de México (Ceballos, G., y G. Oliva, coords.). Comisión Nacional para el Conocimiento y Uso de la Biodiversidad-Fondo de Cultura Económica. Ciudad de México, México.

Arita, H. T. 2005b. Glossophaga leachii (Gray, 1844). Pp. 215-216 en Los mamíferos silvestres de México (Ceballos, G., y G. Oliva, coords.). Comisión Nacional para el Conocimiento y Uso de la Biodiversidad-Fondo de Cultura Económica. Ciudad de México, México.

Brower, J. E., J. H. Zar, y C. N. Von Ende. 1997. Field and laboratory methods for general ecology. WCB-McGraw-Hill. Dubuque, EE.UU.

Cameron, G. N., and S. R. Spencer. 1981. Sigmodon hispidus. Mammalian Species 158:1-9.

Castro-Arellano, I., y G. Ceballos. 2005. Cryptotis mexicana (Coues, 1877). Pp. 133 en Los mamíferos silvestres de México (Ceballos, G., y G. Oliva, coords.). Comisión Nacional para el Conocimiento y Uso de la Biodiversidad-Fondo de Cultura Económica. Ciudad de México, México.

Castro-Arellano, I., y M. Santos. 2005. Heteromys desmarestianus Gray, 1868. Pp. 624-625 en Los mamíferos silvestres de México (Ceballos, G., y G. Oliva, coords.). Comisión Nacional para el Conocimiento y Uso de la Biodiversidad-Fondo de Cultura Económica. Ciudad de México, México.

Ceballos, G. 2005. Spilogale gracilis Merriam, 1890. Pp. 392-393 en Los mamíferos silvestres de México (Ceballos, G., y G. Oliva, coords.). Comisión Nacional para el Conocimiento y Uso de la Biodiversidad-Fondo de Cultura Económica. Ciudad de México, México.

Ceballos, G., J. Arroyo-Cabrales, R. A. Medellín, L. Medrano, y G. Oliva. 2005. Diversidad y Conservación de los mamíferos de México. Pp. 21-49 en Los mamíferos silvestres de México (Ceballos, G., y G. Oliva, coords.). Comisión Nacional para el Conocimiento y Uso de la Biodiversidad-Fondo de Cultura Económica. Ciudad de México, México.

Ceballos, G., y G. Oliva. 2005. Mustela frenata Lichtenstein, 1831. Pp. 380-381 en Los mamíferos silvestres de México (Ceballos, G., y G. Oliva, coords.). Comisión Nacional para el Conocimiento y Uso de la Biodiversidad-Fondo de Cultura Económica. Ciudad de México, México.

Cimé-Pool, J. A., S. F. Hernández-Betancourt, R. C. Barrientos, y A. A. Castro-luna. 2001. Diversidad de pequeños roedores en una selva baja caducifolia espinosa del noreste de Yucatán, México. Therya 1:23-40.

Clench, H. 1979. How to make regional list of butterflies some thoughts. Journal of the Lepidopterists Society 33: 215-231. 
Cruz-Lara, L. E., C. Lorenzo, L. Soto, E. Naranjo, y N. Ramírez. 2004. Diversidad de mamíferos en cafetales y selva mediana de las cañadas de la Selva Lacandona, Chiapas, México. Acta Zoológica Mexicana (n. s.) 20:63-81.

Cuarón, A. D. 2005. Tamandua mexicana (Saussure, 1860). Pp. 121-123 en Los mamíferos silvestres de México (Ceballos, G., y G. Oliva, coords.). Comisión Nacional para el Conocimiento y Uso de la Biodiversidad-Fondo de Cultura Económica. Ciudad de México, México.

Diario Oficial de la Federación. 2010. Norma Oficial Mexicana NOM-059SEMARNAT-2010, Protección ambiental-Especies nativas de México de flora y fauna silvestres-Categorías de riesgo y especificaciones para su inclusión, exclusión o cambio-Lista de especies en riesgo. 30 de diciembre de 2010. Segunda Sección.

Emmons, L. H., y F. Feer. 1997. Neotropical rainforest mammals, a field guide. University of Chicago Press. Chicago, Illinois.

Espinoza-Medinilla, E. E. 2005. Tylomys nudicaudus Peters, 1866. Pp. 807-808 en Los mamíferos silvestres de México (Ceballos, G., y G. Oliva, coords.). Comisión Nacional para el Conocimiento y Uso de la Biodiversidad-Fondo de Cultura Económica. Ciudad de México, México.

Figueroa, F., y H. T. Arita. 2005. Potos flavus (Schreber, 1774). Pp. 406-408 en Los mamíferos silvestres de México (Ceballos, G., y G. Oliva, coords.). Comisión Nacional para el Conocimiento y Uso de la Biodiversidad-Fondo de Cultura Económica. Ciudad de México, México.

Fjeldsa, J. R., C. K. Bowie, y C. Rahbek. 2012. The role of mountain ranges in the diversification of birds. Annual Review of Ecology, Evolution and Systematics 43:249-265.

Fleming, T. H. 1993. Plant-visiting bats. American Scientist 81:460-467.

Gallina, S., A. González-Romero, y R. H. Manson. 2008. Mamíferos pequeños y medianos. Pp. 161-178 in Agroecosistemas cafetaleros deVeracruz: Biodiversidad, Manejo y Conservación (Manson, R. H., V. Hernández-Ortiz, S. Gallina, y K. Mehltreter, eds.). Instituto de Ecología A. C. , Instituto Nacional de Ecología. Ciudad de México, México.

Gálvez-Altúzar, J. 1990. Mastofauna del Parque Nacional Cañón del Sumidero, Chiapas, México. Tesis de Licenciatura. Escuela de Biología, Instituto de Ciencias y Artes de Chiapas. Tuxtla Gutiérrez, México.

García-Estrada, C., A. Damon, C. Sánchez-Hernández, L. Soto, y G. Ibarra. 2006. Bat diversity in montane rainforest and shade coffee under different management regimes in southeastern Chiapas, Mexico. Biological Conservation 132:351-361.

García-Estrada, C., A. Damon, C. Sánchez-Hernández, L. Soto, y G. Ibarra. 2012. Diets of frugivorous bats in montane rainforest and coffee plantations in southeastern Chiapas, Mexico. Biotropica 44:394-401.

García-Estrada, C., M. L. Romero-Almaráz, y C. Sánchez-Hernández. 2002. Comparison of rodent communities in sites with different degrees of disturbance in deciduos forest of Southeastern Morelos, Mexico. Acta Zoológica Mexicana (n. s.) 85:153168. 
Gardner, A. L. 1977. Feeding habits. Pp. 293-350 in Biology of bats of the New World Family Phyllostomidae. Part II (Baker, R. J., J. K., Jhones Jr., and D. C. Carter, eds.). Special publications of the Museum, Texas Tech University 13:1-364.

GaSHWiLer, J. 1959. Small mammal study in best-central Oregon. Journal of mammalogy 40:128-139.

Hall, R. E. 1981. The mammals of North America. John Wiley and Sons. New York, EE.UU.

Halffter, G., y C. Moreno. 2005. Significado biológico de las Diversidades Alfa, Beta y Gamma. Pp. 5-18 in Sobre Diversidad Biológica: El Significado de las Diversidades Alfa, Beta y Gamma (Halffter, G., J. Soberón, P. Koleff, y A. Melic, eds.). Comisión Nacional para el Conocimiento y Uso de la Biodiversidad, Sociedad Entomológica Aragonesa, Grupo Diversitas-México, Consejo Nacional de Ciencia y Tecnología. Zaragoza, España.

Harris, S., y T Woolard. 1990. The dispersal of mammals in agricultural habitats in Britain. Pp. 159-168 in Species dispersal in agricultural habitats (Bunce, R. G. H., y D. C. Howard, eds.). Institute of Terrestrial Ecology, Belhaven Press. London, Reino Unido.

Horváth, A., I. J. March, y J. H. D. Wolf. 2001. Rodent diversity and land use in Montebello, Chiapas, Mexico. Studies on Neotropical Fauna and Environment 36:169-176.

Hurlbert, S. H. 1971. The nonconcept of species diversity: a critique and alternative parameters. Ecology 52: 577-586.

Koleff, P. 2005. Conceptos y medidas de la diversidad beta. Pp. 19-40 en Sobre Diversidad Biológica: El Significado de las Diversidades Alfa, Beta y Gamma (Halffter, G., J. Soberón, P. Koleff, y A. Melic, eds.). Comisión Nacional para el Conocimiento y Uso de la Biodiversidad, Sociedad Entomológica Aragonesa, Grupo Diversitas-México, Consejo Nacional de Ciencia y Tecnología. Zaragoza, España.

López-Arevalo, H., O. Montenegro-Díaz, y A. Cadena. 1993. Ecología de pequeños mamíferos de la Reserva Biológica Carpanta, en la Cordillera Oriental Colombiana. Studies on Neotropical Fauna and Environment 28:193-210.

lópez-Portillo, J. Presidente Constitucional de los Estados Unidos Mexicanos. Secretaría de Asentamientos Humanos y Obras Públicas. 1980. Decreto por el que se declara Parque Nacional, con el nombre de Cañón del Sumidero el área descrita en el considerando quinto, y que se expropia a favor del Gobierno Federal una superficie de 271. 894, 190.00 m2, ubicada en el estado de Chiapas. Diario Oficial de la Federación. 8 de diciembre.

López-Vidal, J. C., y T. Átvarez. 1993. Biología de la Rata Montera, Neotoma mexicana, en La Michilia, Durango, México. Pp. 185-195 en Avances en el estudio de los Mamíferos de México (Medellín, R. A., y G. Ceballos, eds.). Asociación Mexicana de Mastozoología, A. C. Distrito Federal, México.

Llorente, J. A., M. Garcés, T. Pulido, e I. Luna. 1990. Manual de recolecta y preparación de animales. Facultad de Ciencias, Universidad Nacional Autónoma de México. Ciudad de México, México. 
Magurran, A. E. 1989. Ecological Diversity and its Measurement. Ediciones VEDRA. Caspe, Barcelona, España.

Marby, K. E., E. A. Dreelin, y G. W. Barret. 2003. Influence of landscape elements on population densities and habitat use of three small-mammal species. Journal of Mammalogy 84: 20-25.

Medellín, R. A. 1993. Estructura y diversidad de una comunidad de murciélagos en el trópico húmedo mexicano. Pp. 333-354 en Avances en el estudio de los mamíferos de México (Medellín, R. A., y G. Ceballos, eds.). Asociación Mexicana de Mastozoología A. C. Ciudad de México, México.

Medellín, R. A., H. T. Arita, y O. Sánchez. 1997. Identificación de los murciélagos de México, Clave de campo. Asociación Mexicana de Mastozoología, A.C. Ciudad de México, México.

Miranda, F. 1998. La vegetación de Chiapas. Consejo estatal para la cultura y las artes de Chiapas. Tuxtla Gutiérrez, México.

Miranda, F., y E. Hernández X. 1963. Los Tipos de Vegetación de México y su Clasificación. Boletín de la Sociedad Botánica de México 28:29-179.

Moreno, C. E. 2001. Métodos para medir la biodiversidad. Cooperación Iberoamericana Ciencia y Tecnología para el Desarrollo-UNESCO ORCYT-Sociedad Entomológica Aragonesa. Zaragoza, España.

Naranjo, E. J. 2000. Estimación de abundancia y densidad en poblaciones de fauna silvestre tropical. Pp. 37-46 in Manejo de Fauna Silvestre en Amazonia y Latinoamérica (Cabrera, E., C. Mercolli, y R. Resquín, eds.). Fundación Moisés Bertoni y CITES-Paraguay. Asunción, Paraguay.

Naranjo, E. J. 2002. Population ecology and conservation of ungulates in the Lacandon Forest, Mexico. University of Florida. Gainesville, EE.UU.

Naranjo, E. J., C. Lorenzo, y A. Horváth. 2005. La Diversidad de Mamíferos de Chiapas. Pp. 161-193 in Diversidad biológica en Chiapas (González-Espinosa, M., N. Ramírez-Marcial y L. Ruíz-Montoya, coords.). El Colegio de la Frontera Sur-Consejo de Ciencia y Tecnología de Chiapas. Distrito Federal, México.

Nebel, B. J., y R. Wrigth. 1999. Ciencias Ambientales. Ecología y desarrollo sostenible. Editorial Prentice Hall. Distrito Federal, México.

Ovando-Damián, L. 1990. Avifauna del parque nacional cañón del sumidero, Chiapas, México. Tesis de Licenciatura. Escuela de Biología, Instituto de Ciencias y Artes de Chiapas. Tuxtla Gutiérrez, México.

Parmenter, R. R., S. L. Brantley, J. H. Broen, C. S. Crawford, D. C. Lightfott, y T. Yates. 1995. Diversity of animal communities on Southwestern Rangelans: species patterns, habitat relationships and land management. Natural Resources and Environmental Issues 4:50-71.

Pianka, E. R. 1966. Latitudinal gradients in species diversity: a review of concepts. The American Naturalist 100: 33-46.

Primack, R. B. 1998. Essentials of conservation biology. Sinauer, Sunderland. EE.UU.

Pronatura-Chiapas A. C. 2002. Programa de manejo del parque nacional Cañón del Sumidero, Chiapas. San Cristóbal de las Casas, Chipas. Documento Interno. 
Racey, P. A. 1988. Reproductive assessment in bats. Pp. 31-45 in Ecological and behavioural methods for the study of bats (Kunz, T. H., ed.). Smithsonian Institute. Washington, EE.UU.

Ramírez-Pulido, J., C. Aguilar, y A. Castro-Campillo. 2005a. Peromyscus aztecus (Saussure, 1860). Pp. 721-722 en Los mamíferos silvestres de México (Ceballos, G., y G. Oliva, coords.). Comisión Nacional para el Conocimiento y Uso de la Biodiversidad-Fondo de Cultura Económica. Ciudad de México, México.

Ramírez-Pulido, J., J. Arroyo-Cabrales, y A. Castro-Campillo. 2005b. Estado actual y relación nomenclatural de los mamíferos terrestres de México. Acta Zoológica Mexicana (n. s.) 21:21-82.

Ramírez-Pulido, J., y A. Castro-Campillo. 1994. Bibliografía reciente de los mamíferos de México. 1989/1993. Universidad Autónoma Metropolitana. Ciudad de México, México.

Rappole, J. H., D. I. King, y J. H. Vega-Rivera. 2003. Coffe and conservation. Conservation Biology 17:334-336.

Reid, F. A. 1997. A field guide to the mammals of Central America and southeast Mexico. Oxford University Press. New York, EE.UU.

Retana, O., y C. Lorenzo. 2002. Lista de los mamíferos terrestres de Chiapas: endemismos y estado de conservación. Acta Zoológica Mexicana (n. s.) 85:25-49.

Riechers, P. A., y F. Y. De la Cruz. 2012. Roedores en áreas naturales protegidas de Chiapas depositados en la Colección Zoológica Regional Mammalia. Pp. 4757 en Estudios sobre la bilogía de roedores silvestres mexicanos (Cervantes, F. A., y C. Ballesteros-Barrera, eds.). Universidad Nacional Autónoma de MéxicoUniversidad Autónoma Metropolitana. Ciudad de México, México.

Riechers, P. A., M. Martínez-Coronel, y R. Vidal. 2003. Consumo de polen de una colonia de maternidad de Leptonycteris curasoae yerbabuenae en Tuxtla Gutiérrez, Chiapas. Anales del Instituto de Biología, Universidad Nacional Autónoma de México, Serie Zoología 74:46-66.

RieChers, P. A., y R. Vidal. 2009. Registros de Choeronycteris mexicana (Chiroptera: Phyllostomidae) en Chiapas. Revista Mexicana de Biodiversidad 8:879-882.

Silva-López, G. F. García, y E. RodríGuez. 1987. The present status of Ateles and Alouatta in non-extensive forest areas of the Sierra de Santa Marta, Veracruz, Mexico. Primate Conservation 9:53-61.

Soberón, J., y J. Llorente. 1993. The Use of Species Accumulation Function for the Prediction of Species Richness. Conservation Biology 7:480-488.

Sodhi, M. R., C. Posa, T. M. Lee, y I. G. Warkentin. 2008. Effects of disturbances or loss of tropical rainforest on birds. Auk 125:511-519.

Sosa, V. J., E. Hernández-Salazar, D. Hernández-Conrique, y A. Castro-luna. 2008. Murciélagos. Pp. 181-191 en Agroecosistemas cafetaleros de Veracruz: Biodiversidad, Manejo y Conservación (Manson, R. H., V. Hernández-Ortiz, S. Gallina, y K. Mehltreter, eds.). Instituto de Ecología A. C., Instituto Nacional de Ecología . Ciudad de México, México.

SpellerberG, I. F. 1991. Monitoring ecological change. Cambridge University Press. Cambridge, Reino Unido. 
UNEP. 1992. Convention on biological diversity. United Nations Environmental Program, Environmental Law and Institutions Program Activity Centre. Nairobi, Kenia.

Vargas, M., F. A. Cervantes, G. Montiel-Parra, y S. Rubio-Hernández. 2009. Ectosimbiontes de la musaraña Sorex veraecrucis (Mammalia: Soricomorpha) del Estado de Veracruz, México. Pp. 265-270 en 60 años de la Colección Nacional de Mamíferos del Instituto de Biología, UNAM Aportaciones al conocimiento y conservación de los mamíferos mexicanos (Cervantes, F. A., Y. Hortelano y J. Vargas, eds.). Instituto de Biología Universidad Nacional Autónoma de México. Ciudad de México, México.

Vidal-López, R. 1998. Los mamíferos de Coapilla, Chiapas, México. Tesis de Licenciatura. Escuela de Biología, Universidad de Ciencias y Artes de Chiapas. Tuxtla Gutiérrez, México.

Villa, B. 1966. Los Murciélagos de México: su importancia en la economía y la salubridad, su clasificación sistemática. Universidad Autónoma de México, Instituto de Biología. Ciudad de México, México.

Whittaker, R. H. 1972. Evolution and measurement of species diversity. Taxon 21:213251.

Williams-Guillén, K., y I. Perfecto. 2010. Effects of agricultural intensification on the assemblage of leaf-nosed bats (Phyllosotmidae) in a landscape in Chiapas, Mexico. Biotropica 42:605-613.

Zarza, H., y G. Ceballos. 2005. Neotoma mexicana Baird, 1855. Pp. 693-695 en Los mamíferos silvestres de México (Ceballos, G., y G. Oliva, coords.). Comisión Nacional para el Conocimiento y Uso de la Biodiversidad-Fondo de Cultura Económica. Ciudad de México, México.

Sometido: 21 de junio de 2013

Revisado: 10 de septimbre de 2013

Aceptado: 9 de diciembre de 2013

Editor asociado: Jan Schipper

Diseño gráfico editorial: Gerardo Hernández. 